\title{
Self-Determination Theory and Risk Behavior in a Collectivistic Society: Preventing Reckless Driving in Urban Nepal
}

Yerina Ranjit

University of Connecticut - Storrs, yerina.ranjit@uconn.edu

Follow this and additional works at: https://opencommons.uconn.edu/dissertations

\section{Recommended Citation}

Ranjit, Yerina, "Self-Determination Theory and Risk Behavior in a Collectivistic Society: Preventing Reckless Driving in Urban Nepal" (2016). Doctoral Dissertations. 1112.

https://opencommons.uconn.edu/dissertations/1112 
Self-Determination Theory and Risk Behavior in a Collectivistic Society:

Preventing Reckless Driving in Urban Nepal

Yerina S. Ranjit, $\mathrm{PhD}$

University of Connecticut, 2016

\begin{abstract}
Traffic road accidents are one of the leading causes of mortality in Nepal and around the world (WHO, 2011). Drivers in Nepal are not adequately educated about road safety rules, and regulations are also not strictly enforced. Public safety campaigns may be able to alter driver's attitudes and behaviors, however, little is known about which persuasive strategies may be most effective. Drawing on self-determination theory and the health belief model, the current study used a post-only experimental design to test of the impact of collective $v$. individual appeals, and messages that emphasized that the choice to act was up to the participant (autonomy support) $v$. more directive language. Participants were Nepali college students (mean age 20, $N=199$ ). The study found that individualistic messages had a direct impact on safe driving behaviors three weeks after exposure to the messages. Directive messages, rather than autonomy support, led to greater valuing of the point of the message (identified regulation), which in turn increased perceived susceptibility, perceived severity, and behavioral intention. None of the hypothesized moderators changed the relationship between variables. Further implications are discussed.
\end{abstract}


Self-Determination Theory and Risk Behavior in a Collectivistic Society:

Preventing Reckless Driving in Urban Nepal

Yerina S. Ranjit

B.S.W., Nirmala Niketan, College of Social Work, Mumbai, 2000

M.A. Northeastern University, 2008

\author{
A Dissertation \\ Submitted in Partial Fulfillment of the \\ Requirements for the Degree of Philosophy at the \\ University of Connecticut,
}

2016 


\author{
Approval Page \\ Doctor of Philosophy Dissertation
}

\title{
Self-Determination Theory and Risk Behavior in a Collectivistic Society: \\ Preventing Reckless Driving in Urban Nepal
}

Presented by Yerina S. Ranjit, B.S.W., M.A.

Major Advisor

Leslie B. Snyder

Associate Advisor

Mark A. Hamilton

Associate Advisor

Rajiv N. Rimal

University of Connecticut

2016 


\section{Table of Contents}

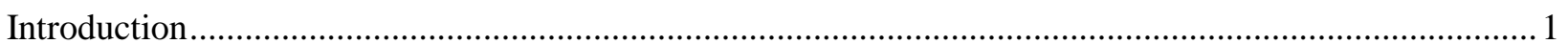

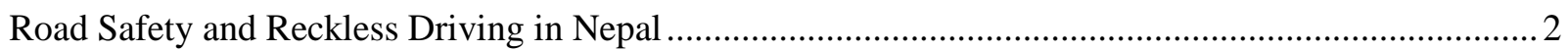

Self-Determination Theory in Explaining Motivations .............................................................................. 3

Autonomy Support Message and Identified Regulation .................................................................

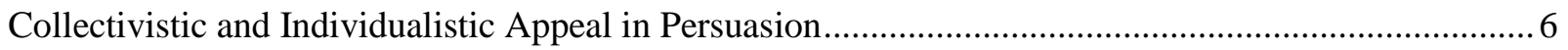

Expansion of Health Belief Model in Risky Behavior...................................................................... 7

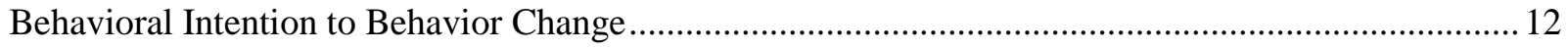

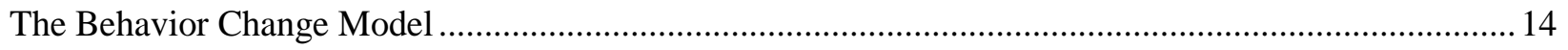

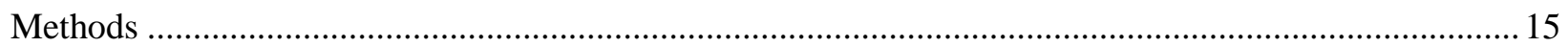

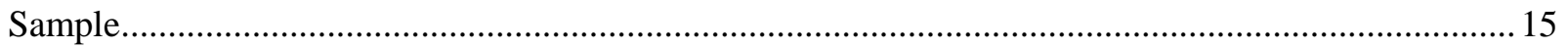

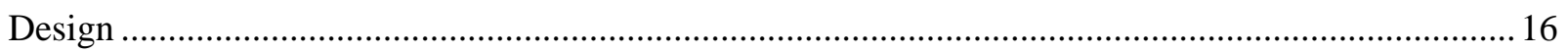

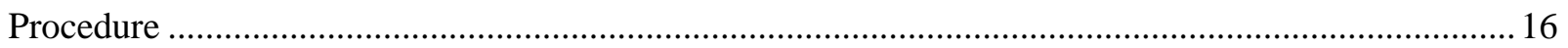

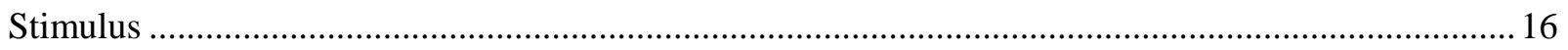

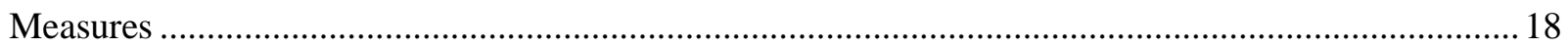

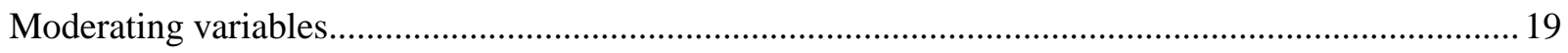

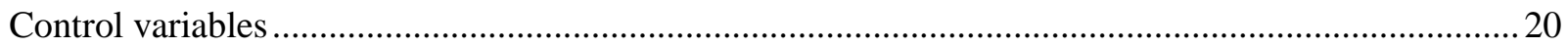

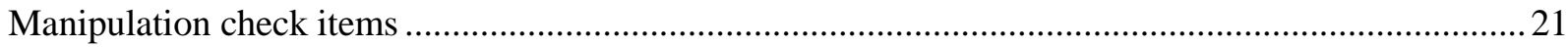

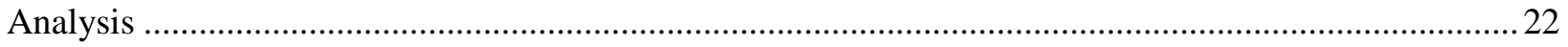

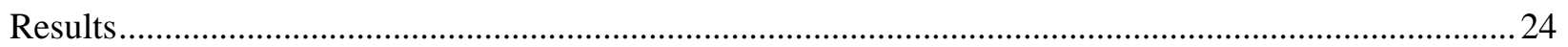

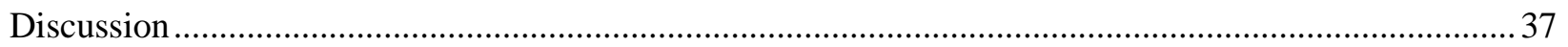

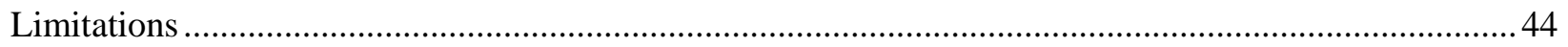

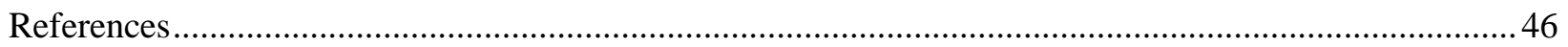




\section{List of Tables and Figures}

Table 1. Logistic regression of variables on participation in the behavioral survey ................. 23

Table 2. Regression of Pretest Measures and Conditions .................................................. 25

Table 3. Summary of Correlations, Means, and Standard Deviations of Variables ................... 27

Table 4. Perceived barriers as moderator of perceived susceptibility and severity on behavioral

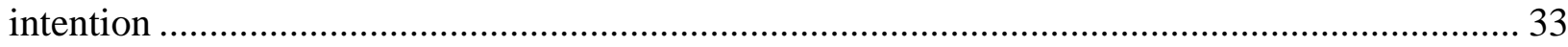

Table 5. Perceived source likability, dynamism, competence and trustworthiness as moderators

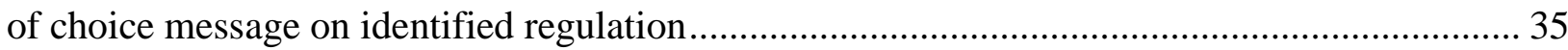

Table 6. Descriptive norm and injunctive norm as moderators of behavioral intention on behavior

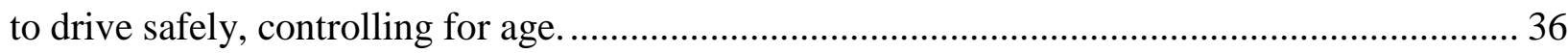

Figure 1. Hypothesized model for predicting behavior using tenets of self-determination theory

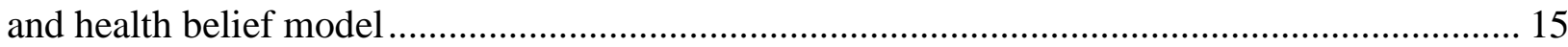

Figure 2. Fit of Hypothesized Path Model Predicting Safe Driving Behavioral Intentions $(\mathrm{N}=$

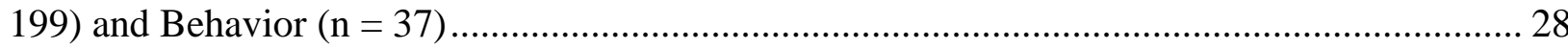

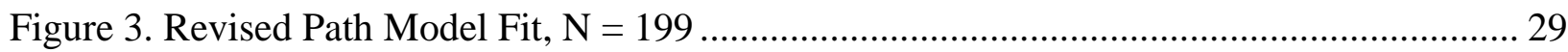

Figure 4. Means of four conditions on behavioral intentions to drive safely ........................... 31

Figure 5. Interaction of conditions on behavioral intention to drive safely ............................. 31 


\section{Self-Determination Theory and Risk Behavior in a Collectivistic Society: \\ Preventing Reckless Driving in Urban Nepal}

\section{Introduction}

Traffic related accidents are one of the leading causes of injuries and deaths in developing countries such as Nepal, which is recognized as an important but neglected public health issue (Franklin, 2013). According to a report published by World Health Organization (2011), globally, traffic accidents account for 1.3 million deaths every year and $90 \%$ of these road accidents occur in middle and low income countries. In Kathmandu, the capital city alone, there are about 130 major accidents every day, which is the main cause of serious life threatening injuries and deaths ("Nepal: Road traffic accidents on the rise," 2012). Additionally, many accidents are related to reckless driving. Accidents reported almost every day involve reckless driving such as passing other vehicles on the wrong side, changing lanes carelessly, speeding, not paying attention to road signage, and not yielding to pedestrians, to name a few (Thapa, 2013). Many of these traffic accidents involve people riding two-wheelers or motorcycles, which are a common mode of transportation in Kathmandu.

Addressing this important public health issue, the current study aims to understand the effect of persuasive messages with a collectivistic appeal in influencing driver's intention to avoid reckless driving, such as unnecessary speeding, and practice road safety. The study also employs the core concept of autonomy support messages proposed by self-determination theory to test its influence and change this unsafe behavior.

This is an important study for both public health and communication scholars, because not only is it the first of the its kind study to be conducted in Nepal with a unique cultural context, but it also makes an important contribution in further understanding the process of 
influence of persuasive messages using tenets of self-determination theory, and addresses this largely overlooked public health problem.

\section{Road Safety and Reckless Driving in Nepal}

Like many developing countries, Nepal's road conditions are not ideal for travelling safely. In the year 2012-2013 alone, there were 13,582 accidents, out of which 11\% were fatal. In the year 2013, nearly 100,000 people died and these deaths have been linked to highway and city road accidents (Pandey, 2013). Most accidents are linked to factors such as poor infrastructure, non-adherence to the traffic rules by drivers and pedestrians, and safety management problems by traffic authority. Reckless driving alone, which includes "negligence by driver" accounted for $44 \%$ of all accidents recorded in 2013 and speeding accounted for $10 \%$ of those accidents (Thapa, 2013).

Looking back at Nepal's transportation history, there were only a few vehicles on the roads till the 1950s (Thapa, 2013). Currently, the city has a high volume of motorcycles, which are considered an affordable means of transportation for the vast majority of the population. There are 1.2 million motorbikes on the roads in Nepal compared to 146,000 cars and 45,000 buses (Thapa, 2013). In the Bagmati zone of the Kathmandu valley, due to fast urbanization and motorization of the city roads, there are 688,028 vehicles registered, out of which $70 \%$ are motorcycles and this number is expected to increase every year making the narrow roads more congested (Urban mobility in Kathmandu: Status and trends). Almost every road accident reported in newspapers involves injury and death of motorcyclists ("Nepal: Road traffic accidents on the rise," 2012; Pandey, 2013; "Valley's traffic unbridled chaos," 2011). A recent death of a young Nepali singer who was on her scooter has brought to public attention the dangerous road conditions for middle class drivers who own motorcycles because of the 
convenience and affordability ("Valley's traffic unbridled chaos," 2011). An epidemiological study of road traffic accidents of Western Nepal found that the majority of the victims of these accidents are young males (85\%) between the ages of 15-30 years old (Mishra, Sinha(Mishra), Shukla, \& Sinha, 2010).

To make matters worse, due to corruption, licenses are issued to drivers without officially passing driving tests, which means that they are not properly trained to operate vehicles. This has increased the number of reckless and amateur drivers on the road (Baharudin \& Pandey, 2014; "Valley's traffic unbridled chaos," 2011). Hence, driving conditions are increasingly dangerous in the city roads and has become a serious public health concern. The causes of this problem are both structural and behavioral. Based on this current situation of road safety in Nepal, this study will focus on three specific individual behavior of reckless driving: speeding, close following and dangerous overtaking (Parker, Manstead, Stradling, Reason, \& Baxter, 1992).

The purpose of this study is to test whether media messages based on the tenets of selfdetermination theory and the health belief model, coupled with the cultural sensitive messages can be used to increase safe driving behaviors.

\section{Self-Determination Theory in Explaining Motivations}

Self-determination theory says that human beings are active and initiate their own behavior (Deci \& Ryan, 1985). People have the innate need to be involved in activities that give them the pleasure and sense of accomplishment just by performing the activity. Intrinsic motivation stems from engaging in an activity for the pleasure of performing the activity itself and that is void of any external pressure. Participating in intrinsically interesting activity fulfills one's

psychological needs for competence, autonomy and relatedness (Deci \& Ryan, 1985, 2012; Ryan \& Deci, 2000). However, in the practical world, people do not always have the opportunity to 
engage in behavior that they find genuinely interesting. Self-determination theory posits that living within the boundaries of society, individuals often engage in activities out of compulsion, competition or the desire to please others (Ryan \& Deci, 2000). Some external factors that drive human behavior are monetary or material gain, need to conform to norms, threats of punishments, deadlines, etc. These external pressures are termed extrinsic motivation (Deci \& Ryan, 1985).

Extrinsic motivation. There are many behaviors that are recommended to individuals and imposed by society for positive outcome for the individual and for smooth functioning of the society. For example, exercising or eating healthy may not be an act of enjoyment and intrinsically motivating. However, those who follow these healthy behaviors may choose to do so for reasons such as to gain recognition from friends, to comply with authority, or to gain rewards attached to undertaking the action (Ryan \& Deci, 2000). These non-intrinsic or extrinsically motivated behaviors are defined as "performance of an activity in order to attain some separable outcome" (Ryan \& Deci, 2000, p. 71).

The organismic integration theory, a sub-theory within self-determination theory explains the different types of extrinsic motivations (Deci \& Ryan, 1985). A system of tangible rewards and punishment, such as material gain or avoiding deprivation of those, can effectively control behavior. These pressures influence external regulations of an individual. Less tangible control mechanism of social norms also influence behavior change through introjected regulation. When an individual tries to live up to the social expectations to maintain one's social status or selfesteem, the behavior change is still viewed as caused by external factors. The locus of causality for these behaviors is perceived to be external. The organismic integration theory suggests that there are other forms of extrinsic motivations that influence behavior. These external demands 
motivate individuals to change behavior by helping them see value in performing the behavior. This is termed identified regulation, and the type of motivation that individuals integrate in self during the process of socialization and enact them as if they were an integral part of their belief system is integrated regulation (Deci \& Ryan, 2012; Ryan \& Deci, 2000).

These types of extrinsic motivation that are optimum for sustained behavior change because individuals internalize expectations and perceive the self as the locus of causality of the behavior (Ryan \& Deci, 2000). Individuals perceive the cause of the behavior to be within self when the behavior is perceived to be initiated and performed out of the individual's choice, and not because of an external pressure. This perception increases the sense of autonomy.

\section{Autonomy Support Message and Identified Regulation}

Autonomy-support refers to "taking the others' perspective, encouraging initiation and exploration, providing choice, and being responsive to others" (Deci \& Ryan, 2012, p. 422). This is a supportive environment where individuals are directed to a certain goal by giving individuals a sense of freedom of choice, encouragement, and a system of feedback (Deci \& Ryan, 2012). The theory thus posits that autonomy-supportive communication encourages individuals to identify with the recommended behavior.

The idea of autonomy-support thus assumes that if an individual does not perform an action by seeing the value in it, the behavior change is not sustained. Identified regulation gives the individual a sense of satisfaction, and contributes to the individual's psychological wellbeing, which stems from a nurturing environment that fulfills the individual's psychological needs for competence, autonomy and relatedness (Ryan \& Deci, 2000). This positive environment that facilitates individuals' satisfaction stems from autonomy-support environment. 
Hence, this study will attempt to establish the role of identified regulation as the cognitive process of behavior change in the context of health messages that incorporates autonomy-support language. Previous public health studies have shown that autonomy-support communication or messages that highlight the individual's ability to make a choice for himself or herself positively influences their sense of identified regulation (termed as "choice message" henceforth), which will then influence the intention to take a certain action (Chatzisarantis, Hagger, Biddle, Smith, \& Wang, 2003; Williams, Niemiec, Patrick, Ryan, \& Deci, 2009) . Thus the following hypothesis is proposed:

H1: Choice message will be positively related to identified regulation.

\section{Collectivistic and Individualistic Appeal in Persuasion}

Geert Hofstede's classical dimension of cultures classify Asian cultures as collectivistic, where individuals focus on interdependence, family security, harmony, cooperation, social hierarchies (Hofstede, 2001). Whereas in individualistic cultures, the emphasis is on independence, personal achievement, freedom, high levels of competition and pleasure. Research shows that belonging to either a collectivistic or individualistic culture influences the way persuasive messages are processed. Studies have shown that messages that highlight interdependence are more effective in collectivistic cultures, and conversely, messages that focus on self are more effective in individualistic cultures (S.-P. Han \& Shavitt, 1994; Jing, 2010; Khaled, Biddle, Noble, Barr, \& Fischer, 2006). The S.-P. Han and Shavitt (1994) study showed that in Korean culture, advertisement with collectivistic appeal that focused on group integrity and group well-being was much more effective than they were in the individualistic culture of United States where individualistic appeal that focused on self-improvement and benefit to the self was more effective on choosing a product. Nepal is largely a collectivistic society, where 
members of family and extended family play an important role in everyday life. Elders and people with authority are supposed to be respected, and making other people who are important to an individual happy is a key motivator for behavior (Hofstede, 2001). Given this cultural background, this study posits that collectivistic appeal message will have a stronger influence on behavioral intention than individualistic appeal. In case of reckless driving, collectivistic appeal message will constitute of reducing harm to others, and individualistic appeal message will constitute of a message focuses on the reduction of harm to self. Hence, this study predicts that collectivistic appeal message will influence one's behavioral intention:

$\mathrm{H} 2$ : Collectivistic appeal message will have a stronger positive influence on behavioral intention than individualistic appeal message.

Based on the above hypotheses, the study further proposes that when messages provide both collectivistic appeal and a sense of autonomy support with strong choice wordings, then it will influence one's intention to adopt the recommended behavior. Hence, the following hypothesis is proposed:

H3: A crossed message of strong choice message and collectivistic message will be directly related to behavioral intention.

\section{Expansion of Health Belief Model in Risky Behavior}

This study proposes a theoretical model based on the tenets of the Health Belief Model $(\mathrm{HBM})$ in an attempt to elaborate the process that influences health decision making - in this case, the intention to drive safely. The Health Belief Model proposed by Rosenstock (1966) has been associated with predicting various positive health behaviors (Champion \& Skinner, 2008a; Janz \& Becker, 1984). The main tenets of the HBM are perceived susceptibility, perceived 
severity, perceived benefits, perceived barriers and cues to action (Janz \& Becker, 1984;

Carpenter, 2010).

Perceived susceptibility. Perceived susceptibility has been defined as one's "belief about the chances of experiencing a risk or getting a condition or disease" (Champion \& Skinner, 2008a, p. 48). In this case, it is motorcycle riders' belief that they are at risk of getting injured, experiencing life of dependency and even death if they drive recklessly. It's one's subjective perception of how vulnerable one is to experiencing serious health concerns due to a traffic accident.

Perceived severity. Perceived severity, on the other hand, is a person's perception of seriousness of the condition associated with the outcome of an unsafe behavior or a health condition, such as death, physical impairment, pain, to name a few (Janz \& Becker, 1984). The two concepts of perceived susceptibility and perceived severity is also an integral part of another health communication theory - the Extended Parallel Process Model (EPPM) - which has been employed in understanding fear appeals in health messages such as smoking cessation, drunk driving and other risky behavior (Witte, 1992; Witte, 1994). In the Extended Parallel Process Model treats perceived threat as an additive index of the two concepts. Other models such as protection motivation theory also treats perceived susceptibility and perceived severity as an additive index of threat, while risk perception study has used a multiplicative index of perceived susceptibility and perceived severity to represent risk perception (Rimal \& Real, 2003a). ElToukhy (2015) made a clear distinction between perceived susceptibility and perceived severity. Perception of susceptibility is a subjective expectation related to an outcome, which is conceptualized as perceived severity. The study demonstrated that these two concepts are rather inversely related to each other, and that when individuals perceived of the prevalence of a certain 
condition to be high, their perceived susceptibility increased while their perceived severity decreased. Because there is still contention among researchers about the threat as a higher order factor, this study will treat perceived severity and susceptibility as two separate concepts and enter into the proposed model.

The Health Belief Model posits that perceived susceptibility and perceived severity are predictive of one's intention to avoid the threat and therefore adopt the recommended behavior (Janz \& Becker, 1984). In the case of motorcycle riders exposed to the health messages, the perception of one's risk or vulnerability to negative health outcome, and the perception of the seriousness of that threat should trigger the intention to drive safely. In an effort to further elaborate the process of behavior change, this study proposes the following relationships: identified regulation will positively influence perceived susceptibility and perceived severity, which then influences behavioral intention to ride safely. Based on this assumption, the following hypotheses are proposed:

H4: Identified regulation will be positively related to perceived susceptibility. H5: Identified regulation will be positively related to perceived severity. H6: Perceived susceptibility will be positively related to behavioral intention H7: Perceived severity will be positively related to behavioral intention.

Perceived barrier. Perceived barriers has been defined as "potential negative aspects of a particular health action" (Janz \& Becker, 1984, p. 2). These barriers to action may be perceived or real. Expense, an unpleasant experience, pain, inconvenience, inaccessibility are some reasons why an individual may perceive the recommended behavior as more costly compared to the benefits associated with the health behavior. Thus, even when an individual acknowledges and understands the value of driving safely, he or she may continue to do so because of certain 
perceived barriers to achieving that goal. In the context of the traffic condition of Kathmandu, external factors beyond one's control such as, an individual's lack of knowledge or understanding of traffic rules, reckless behavior of fellow drivers, unsafe physical road conditions and lack of proper enforcement of traffic may be perceived as barriers to safe driving. Although the health belief model traditionally treats perceived barriers as a mediator of threat effects on behavior, we predict that it will act as a moderator, explaining at least in part the conditions under which threats have an impact on behavior:

H8: Perceived barriers will moderate the relationship between perceived susceptibility and behavioral intention, such that when perceived barriers are high, the relationship between perceived susceptibility and behavioral intention will be weak, and when perceived barriers are low, the relationship between perceived threat and behavioral intention will be strong.

H9: Perceived barriers will moderate the relationship between perceived severity and behavioral intention, such that when perceived barriers are high, the relationship between perceived severity and behavioral intention will be weak, and when perceived barriers are low, the relationship between perceived threat and behavioral intention will be strong.

Source credibility. According to the information processing theory, the evaluation of the source will influence the effectiveness or perceived credibility of the message (Hass, 1981; Hovland \& Weiss, 1951; Pornpitakpan, 2004). When exposed to a message, a receiver will evaluate the credibility of the source based on the source's appearance and demographic information available to the receiver. Credibility has been defined as "reasonable grounds for being believed" (Meyer, 1988, p. 567). This source characteristic has also been defined as the "source's expertise on the topic and trustworthiness as a communicator" (Hass, 1981, p. 143).Source credibility influences the intended persuasive effect of the message (Burgoon et al., 
2000; Hovland \& Weiss, 1951). This study posits that autonomy support in the form of a freedom-of--choice message will influence an individual's identified regulation. In other words, when an individual is exposed to a message that highlights his or her ability to make the right choice for themselves, the individual is more likely to perceive the message positively and see value in the recommended behavior than an individual exposed to messages that seemingly deprive them of the ability to make a choice. However, if the perceived credibility of the source is low, the message may not have the intended effect on the recipient of the message. Hence, this study posits that perceived source credibility will moderate the relationship between exposure to a freedom-of-choice message and identified regulation.

The dimensions of source credibility can be categorized into source dynamism, likability, trustworthiness and source competence (Hass, 1981).In the context of Nepali culture, which is highly patriarchal and is marked by high power distance where people demand and are given respect because of age, an older male can generate perception of source credibility. This study will aim to examine each of the components of perceived source credibility as moderators that will alter the degree of influence of identified regulations upon exposure to the message. The study hypothesizes that upon exposure to the message, the perception of source dynamism, likability, trust worthiness and competence will moderate the perception of identified regulations. Since it is unclear which component of source credibility will be more important in the particular context of the current study, all four components will be tested.

H10: Perceived source dynamism will moderate the relationship between choice message and identified regulation, such that when perceived source dynamism is high, the relationship between choice message and identified regulation will be strong, and when perceived source 
dynamism is low, the relationship between choice message and identified regulation will be weak.

H11: Perceived source likability will moderate the relationship between choice message and identified regulation, such that when perceived source likability is high, the relationship between choice message and identified regulation will be strong, and when perceived source likability is low, the relationship between choice message and identified regulation will be weak.

H12: Perceived source trust worthiness will moderate the relationship between choice message and identified regulation, such that when perceived source trust worthiness is high, the relationship between choice message and identified regulation will be strong, and when perceived source trust worthiness is low, the relationship between choice message and identified regulation will be weak.

H13: Perceived source competence will moderate the relationship between choice message and identified regulation, such that when perceived source expertise is high, the relationship between choice message and identified regulation will be strong, and when perceived source expertise is low, the relationship between choice message and identified regulation will be weak

\section{Behavioral Intention to Behavior Change}

Behavioral intention has been defined as "self-instructions" to carry out a certain behavior (Webb \& Sheeran, 2006, p. 249). Meta-analytical research has established that behavioral change, when measured in a short interval of time, is correlated with and predicted by behavioral intention (Webb \& Sheeran, 2006). Theories such as theory of reasoned action and theory of planned behavior look at intention as a precursor to behavior (Ajzen \& Fishbein, 1980). However, factors such as perceived social pressure to perform the behavior or to avoid it, 
habitual control, self-monitoring, self-efficacy, perceived control over behavior moderate the relationship between intention and actual behavior change (Ajzen \& Madden, 1986; Rivis \& Sheeran, 2003; Webb \& Sheeran, 2006; Wong \& Sheth, 1985). Since this study will be conducted in a collectivistic culture, social norm, especially, both descriptive norm or the perceived prevalence of the behavior, and injunctive norm, or the perceived approval of people who are close to the individual (Cialdini, 1994, 2001; Rimal \& Real, 2003b), will influence one's behavior despite intentions. Since this is a collectivistic culture where people are highly concerned about the opinions of relatives, neighbors and even acquaintances, this study proposes that a sense of "society" consisting of people in these networks, is also an important part that contribute to one's sense of injunctive norm. Thus, the following hypotheses are proposed:

H14: Behavioral intention will be positively related to behavior.

H15: The relationship between behavioral intention and behavior will be moderated by descriptive norm, such that when descriptive norm is high, the relationship between behavioral intention and behavior will be weak, and when descriptive norm is low, the relationship between behavioral intention and behavior will be strong.

H16: The relationship between behavioral intention and behavior will be moderated by injunctive norm, such that when injunctive norm is high, the relationship between behavioral intention and behavior will be weak, and when injunctive norm is low, the relationship between behavioral intention and behavior will be strong.

Participants of this study are college students. Young adults of this age group generally are not concerned with the approval of important others in their lives such as parents or professors (Gibbons, Gerrard, \& Lane, 2003). Literature on norms suggests that people of this age group are more influenced by how prevalent the behavior is among their peers. Based on 
this, this study posits that descriptive norms will be a stronger moderator between behavioral intention and behavior.

Note that the relative weighting of descriptive and injunctive norms has not been tested before with young urban adults in a collectivist society.

\section{The Behavior Change Model}

Implicit in the hypothesis above is a model of behavior changed that draws on both the health belief model and self-determination theory. The model adds identified regulation from self-determination theory to the process of influence from message exposure to behavioral intention (see Figure 1). Perception of threat is a cognitive process and internal regulations, such as identified regulation is an emotional process where an individual feels satisfied in performing the recommended behavior. This study hence adds the component of emotional response to the message, which will mediate the path from exposure to perceived threat.

Adding the elements of messages and elaborating the components of source credibility, the following expanded model demonstrates the process of behavior change.

Figure 1. Hypothesized model for predicting behavior using tenets of self-determination theory and health belief model 
Figure 1. Hypothesized model for predicting behavior using tenets of self-determination theory and health belief model

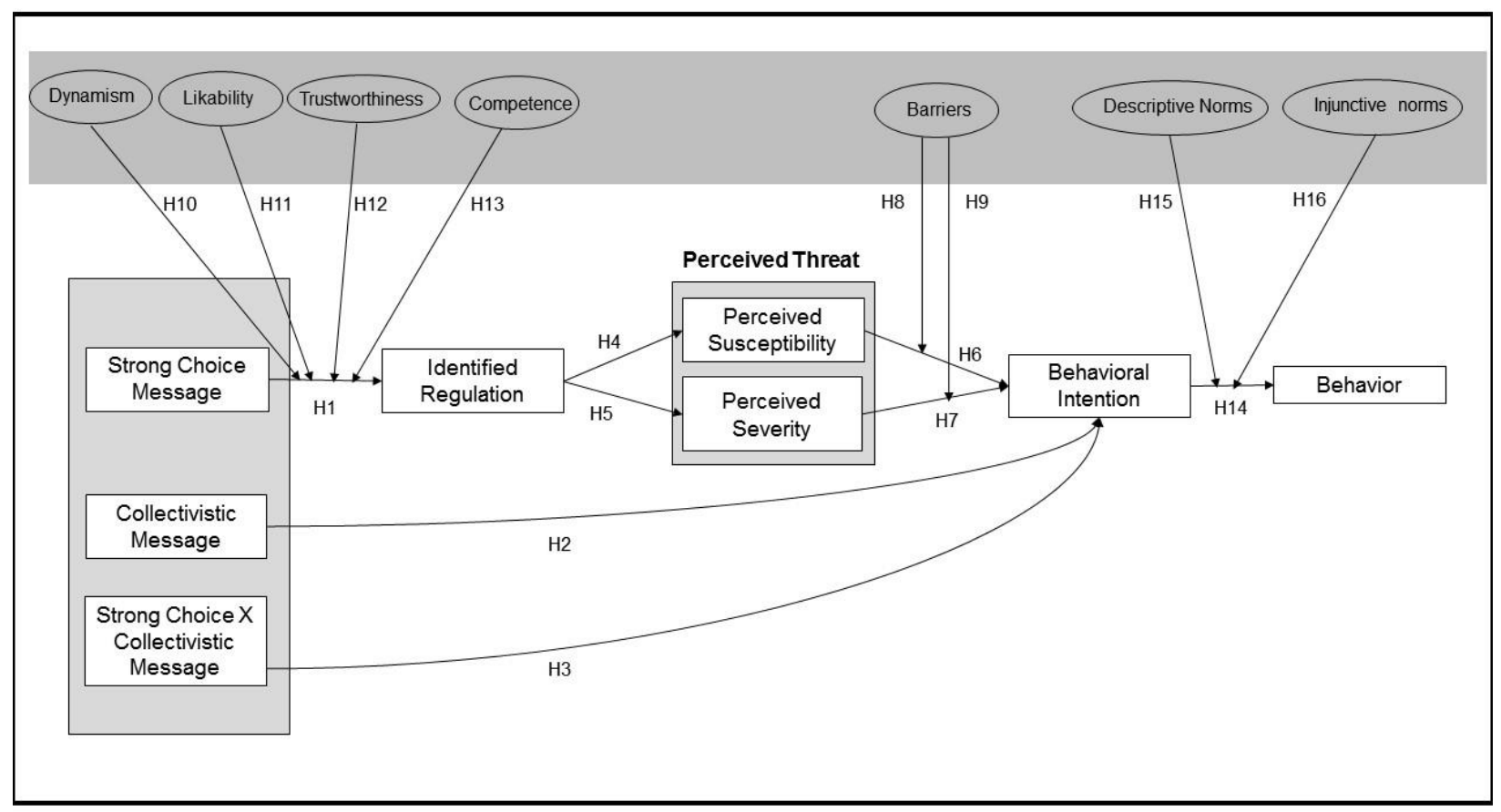

\section{Methods}

\section{Sample}

College students were recruited from eight different private and public colleges in

Kathmandu, Nepal $(\mathrm{N}=199)$. The participants were $75.4 \%$ male. The average age was 20.9 years. On an average, the participants had been riding a motorcycle for the duration of about three years. The survey was taken in two languages (Nepali and English) and 63\% of the participant filled them out in English. An independent samples t-test on variables showed that there was no significant difference between the two language groups. Hence, the groups were not treated differently. 


\section{Design}

This study used a post-only 2 × 2 factorial design with participants randomly assigned to conditions. The factors were strong choice message vs. weak choice message, and individualistic appeal vs. collectivistic appeal messages.

Procedure

Pretest. A pretest was conducted to see if the videos had the intended effect. In the sample $(n=78)$, participants were randomly assigned to each of the four conditions and a survey questionnaire was administered after they watched the videos.

Main study. For main study, participants were recruited with the help of college administrators who made announcements in classrooms asking students who rode motorcycles to participate in the experiment in-between classes. The researcher set up the experiment in a location (empty classrooms) assigned by the college administrator. After random assignment to one of the four conditions, participants viewed the experimental videos on separate computers. Then posttest paper and pencil questionnaire was administered. Participants were asked for their e-mail address at the end of the experiment for the follow up survey.

A post-test survey of behavior was administered after three weeks by emailing a survey link (and three reminders all one week apart) to all participants.

\section{Stimulus}

A professional documentary film producer and video editor produced a video lasting about one minute for each of the four conditions. The video featured a Nepali male voice over who gave messages in Nepali language.

All messages included the same information regarding driving safely (See Appendix 2 for detailed stimuli). Three behaviors were recommended for safe driving: "Avoid speeding, 
especially at night when the roads are not well lit"; "Avoid dangerous overtaking, especially when you cannot see properly what's ahead of you"; and "Avoid driving too close to the vehicle in front of you" (Parker et al., 1992). All conditions saw the same the vivid video of an accident - a widely viewed viral online YouTube video clip of an actual event captured by a street camera at an intersection in the capital city (Kathmandu) that shows a bus running over a motorcyclist). All the videos were of the same length of 1 minute 49 seconds.

The key messages per condition appeared as text on the screen and was heard as a voiceover. The strong choice message included the words, "It is in your hands to avoid traffic mishaps. It is up to you to choose to drive carefully. You are capable of making the right choice for yourself." The weak choice message, on the other hand, was a directive and did not emphasize the autonomy of the driver. The message was, "You should not drive recklessly. You should follow the traffic rules. Stop driving recklessly". The collectivistic appeal message read, "Driving recklessly can have severe consequences. It can lead others to lose their limbs and live a life of dependency. Not driving recklessly will keep the roads safe, and you could be saving someone's son, daughter, mother, father or a relative." And the individualistic appeal message read, "Driving recklessly can have severe consequences. It can lead you to lose your limbs and live a life of dependency. You could live a long and healthy life if you drive carefully and you could also be saving yourself from being caught by the traffic police." 


\section{Measures}

The survey measures used were administered in Nepali or English based on the preference of language of the participants. A native Nepali speaker, a graduate student at a U.S. university also fluent in English translated the measurement tool.

A Confirmatory Factor Analysis (CFA) was conducted for internal consistency and parallelism of each of the variables using CFA10 (Hamilton, 2002). Items that did not load primarily on the factor or loaded less than .50 were removed from the factor to create the variables.

Behavioral intention. Behavioral intention to drive safely was measured by an additive index of three items: In the next 15 days, how likely are you to speed?; In the next 15 days, how likely are you to drive closely behind another vehicle?; In the next 15 days, how likely are you to overtake dangerously, even when you cannot see clearly what is ahead of you? Each item was assessed on a 5 point bi-polar scale of (1) Not Likely At All to (5) Highly Likely $(M=3.77, S D=$ $.88, \alpha=.80$ ). The variable was reversed to reflect participants' intention to drive safely (Rimal, Lapinski, Cook, \& Real, 2005).

Behavior. The behavior of driving safely was measured using three items: In the past month, I drove my motorcycle in speed; In the past month, I drove my motorcycle close to the vehicle in front of me; In the past month, I dangerously overtook while I was driving my motorcycle. These items were measured on a 5 point Likert scale (1) Strongly Disagree to (5) Strongly Agree $(M=3.26, S D=.89, N=37, \alpha=.70)$. Each item was reversed to reflect the participants' intention to drive safely.

Identified regulation. This scale was adapted from Treatment Self-Regulation Questionnaire (TSRQ) developed by Ryan and Connell (1989). Identified regulation was 
measured using six items. The stem question was about all three behaviors: "The reason I would not speed/overtake dangerously/ drive too close to another vehicle would be..." and the responses were: Because I feel that I want to take responsibility for myself; Because I have carefully thought about it and believe it is very important for many aspects of my life; Because it is consistent with my life goals; Because I personally believe it is the best thing for me; Because it is an important choice I really want to make, and; Because it is very important for being as healthy and safe as possible on a 5 point Likert scale (1) Strongly Disagree to (5) Strongly Agree $(M=4.27, S D=.54, N=199, \alpha=.78)$ (Williams, Rodin, Ryan, Grolnick, \& Deci, 1998). Perceived susceptibility. Perceived susceptibility consisted of three 5 point Likert scale items: I may have serious problems if I drive in speed; The chances of me getting into trouble if I overtake when I cannot see the oncoming traffic is high; I am at risk of getting into accidents due to speeding $(M=4.38, S D=.61, \alpha=.70)(J a n z \&$ Becker, 1984; Witte, Cameron, McKeon, \& Berkowitz, 1996).

Perceived severity. Perceived severity was measured using four items: Overtaking dangerously can lead to severe outcomes; Driving too close to another vehicle is harmful; Driving too close to another vehicle is risky and; Driving in speed leads to bad consequences. The items were assessed on a 5 point Likert scale (1) Strongly Disagree to (5) Strongly Agree (M $=4.31, S D=.54, \alpha=.68)($ Janz \& Becker, 1984; Witte et al., 1996).

Moderating variables

Perceived source likeability. Perceived source likeability was measured using three items -- The source of this message is likable; The source of this message is nice; The source of this message is pleasant -- on a 5 point Likert scale (1) Strongly Disagree to (5) Strongly Agree $(M=$ 3.99, $S D=.61, \alpha=.50)($ Hass, 1981). 
Perceived source dynamism. Perceived source dynamism was measured using two 5 point Likert scale- items: The source of this information is dynamic; and The source of this message is expressive $(M=3.84, S D=.61, \alpha=.58)$ (Hass, 1981).

Perceived source competence. Perceived source competence was measured using three 5 point Likert scale items: The source of this message is educated; The source of this message knowledgeable; The source of this message is not ignorant $(M=4.13, S D=.61, \alpha=.68)$ (Hass, 1981).

Perceived source trustworthiness. Perceived source trustworthiness was measured using three items: The source of this message is trustworthy; I have confidence in the sources advice; The source of this message is honest on a 5 point Likert scale (1) Strongly Disagree to (5) Strongly Agree $(M=4.10, S D=.63, \alpha=.76)($ Hass, 1981).

Injunctive norm. Injunctive norm was measured on a bipolar scale using three items. The stems were: Society views speeding as; Society views overtaking when you cannot see what's ahead of you as; and Society views driving too close to another vehicle as, and the response was a bipolar scale of unfavorable to favorable $(M=1.85, S D=.89 \alpha=.82)$ (Rimal \& Real, 2003b).

Descriptive norm. Descriptive norm consisted of an additive scale of four 5 point Likert items: Most people in Kathmandu speed; Most people in Kathmandu drive very close to another vehicle; Most drivers on the roads of Kathmandu overtake even when they cannot see clearly what's ahead of them; Most people of my age overtake dangerously $(M=4.38, S D=.47, \alpha=.60)$ (Klein \& Boster, 2006).

\section{Control variables}

Perceived barriers. Perceived barriers to driving safely was assessed using a two item additive index. The 5 point Likert scale items were: I do not understand traffic rules; I do not 
understand the traffic signs posted on the road $(M=1.87, S D=.68, \alpha=.66)$ (Janz \& Becker, 1984).

Reactance. Psychological reactance was measured using three 5 point Likert scale items combined into an additive scale. The items were: It irritates me that this Public Service Announcement told me how to feel about driving safely; I don't like that I am being told how to feel about driving safely; I dislike that I am being told how to feel about driving safely $(M=2.02$, $S D=.66, \alpha=.76)$ (Donnell, Thomas, \& Buboltz Jr, 2001).

Demographic information. Age, sex, length of time they had been riding motorbikes, and past behavior were measured.

Past behavior. Past behavior was measured using four 5 point Likert scale items combined into an additive index. The items were: I speed in my motorcycle once in a while; I overtake dangerously on my motorbike once in a while; I speed whenever I see that the road is empty; and I habitually drive too close to other vehicles on the road $(M=3.38, S D=.67, \alpha$ $=.68)$.

\section{Manipulation check items}

Perception of autonomy-support (Strong Choice). Perception of autonomy-support was measured using three items, namely: This message talks about individual's ability to make their own choice; This message talks about individual's ability to make the right choice; and This message talks about how an individual's ability to make a decision is up to himself or herself on a 5 point Likert scale (1) Strongly Disagree to (5) Strongly Agree ( $\mathrm{M}=3.83, \mathrm{SD}=.81, \mathrm{n}=78, \alpha$ $=.62)$.

Perception of control (Weak Choice). Weak choice was measured using three items, namely: This message tells you what you should and should not do; This message makes you feel 
as if you are being controlled; This message makes you feel as if how you behave is not your choice. However, this was a very poor scale with low internal consistency and reliability ( $\alpha$ $=.04)$. Hence this scale was not used for further analysis.

Individualistic appeal. Individualistic appeal was measured with five items, namely: This message talks about how you yourself affected by your speeding, This message talks about how you yourself will be affected by your dangerously overtaking, This message talks about how you yourself will be affected by your driving too close to another vehicle, This message talks about how you and your health will be affected by your reckless driving; and The message talks about the consequences of speeding/dangerously overtaking/driving too close to another vehicle on yourself $(\mathrm{M}=4.34, \mathrm{SD}=.43, \mathrm{n}=78, \alpha=.63)$.

Collectivistic appeal. Collectivistic appeal was measured using four items, namely: The message talks about the consequences of reckless driving on others, This message talks about how other people will be affected by your speeding, This message talks about how other people will be affected by your dangerously overtaking, and This message talks about how other people will be affected by your driving too close to another vehicle $(\mathrm{M}=4.18, \mathrm{SD}=.71, \mathrm{n}=78, \alpha=.81)$.

\section{Analysis}

The scales used to create the variables were tested using confirmatory factor analysis. The manipulations were tested using measures of autonomy-support, collective appeal and individualistic appeal that were measured in a pretest of the stimuli $(N=78)$. For the main study $(N=199)$, path analysis was conducted to test hypothesized model, including the mediated relationships in the model using PATH 6.0 (Hunter \& Hamilton, 2002) (H1 - H7, and H14 in Figure 1). The analysis for behavior had a significant decrease in the population size, from 197 to 37, due to attrition. 
To test moderating relationships (H8-H13, H15-16), dependent variables were regressed on the independent variable, moderating variable, and a multiplicative term composed of the $\mathrm{z}$ score of the independent variable multiplied by the z-score of the moderating variable (Fairchild \& MacKinnon, 2009). If moderation was found, additional regression analyses determined the nature of the interaction. Two groups were created using a median split on the moderating variable, and regressions were conducted for each group.

Unfortunately, only 37 out of 199 participated in the 3-week post-test survey. Therefore, it was appropriate to investigate whether participation was biased. A binary logistical regression was conducted, with participation dummy coded 0 for non-participation and 1 for participation. The independent variables were: past behavior, perceived barriers, reactance and age. Out of the variables measured at time one, only age of participants $(\beta=.17, p=.027$; OR $=1.19)$ predicted non-participation (see Table 1). Given this bias, age was controlled for in the rest of the analysis.

Table 1. Logistic regression of variables on participation in the behavioral survey

\begin{tabular}{lccccccc}
\hline \hline & \multicolumn{3}{c}{ Wald's } & & & $e^{\beta}$ \\
Independent Variables & $\beta$ & SE $(\beta)$ & $\chi^{2}$ & $d f$ & $p$ & (odds ratio) \\
\hline Reactance & -.588 & .338 & 3.024 & 1 & .082 & .556 \\
Age & .171 & .077 & 4.888 & 1 & .027 & 1.186 \\
Sex & .127 & .456 & .077 & 1 & .781 & 1.135 \\
Perceived barriers & -.086 & .286 & .091 & 1 & .763 & .917 \\
Past behavior & .273 & .297 & .844 & 1 & .358 & 1.314 \\
Constant & -4.774 & 2.213 & 4.654 & 1 & .031 & .008 \\
\hline \hline
\end{tabular}

Note. The dependent variable in this analysis is participation in survey after three weeks of exposure to stimuli. Non-participation is coded 0 and participation is coded 1 . $\mathrm{N}=199$. 
The path model, which was based on a correlation matrix, used pairwise deletion of missing values to preserve the larger sample size for most of the relationships in the model. The path and moderator analyses predicting behavior change had only 37 participants.

\section{Results}

Manipulation check. To conduct the manipulation check, respondent's perceptions of the content of the message - in terms of autonomy support, individual appeal, and collective appeal - were compared to the assigned conditions. Perception of autonomy-support was regressed on whether or not a person was in one of the two choice conditions. However, exposure to a choice message did not significantly predict perceptions that the message contained information about autonomy support. Thus, the autonomy-support manipulation failed; the strong choice message was not seen as more supportive than the weak choice message.

Two variables were used to test the manipulation of collective vs individual appeals. Being in the collectivistic condition was inversely related to perceiving an individualistic appeal $(\beta=-.32, p=.004, n=77)$. At the same time, being in the collectivistic condition was not significantly related to perceiving a collectivistic appeal (see Table 2). Since those who viewed an individualistic appeal perceived it to be more individualistic than the collective appeal, the manipulation was seen as successful.

Unfortunately, it was not possible to alter the messages after they were produced. The original videos from the pretest were used in the main study. 
Table 2. Regression of Pretest Measures and Conditions

\begin{tabular}{|c|c|c|c|c|c|}
\hline & \multicolumn{4}{|c|}{ Perceived Autonomy support (Choice Message) } & \multirow[b]{2}{*}{$p$ (Sig.) } \\
\hline & $B$ & $S E$ & $B$ & $t$ & \\
\hline$\overline{\text { (Constant) }}$ & 3.833 & .494 & & 41.169 & .000 \\
\hline \multirow[t]{2}{*}{ 1. Choice condition } & .058 & .093 & .071 & .622 & .536 \\
\hline & \multicolumn{3}{|c|}{ Collectivist Appeal } & & \\
\hline (Constant) & 4.186 & .080 & & 52.223 & .000 \\
\hline \multirow[t]{2}{*}{ 1. Collective condition } & .063 & .080 & .089 & .783 & .436 \\
\hline & \multicolumn{3}{|c|}{ Individualistic Appeal } & & \\
\hline (Constant) & 4.339 & .047 & & 92.133 & .000 \\
\hline 1. Collective condition & -.139 & .047 & -.321 & -2.951 & .004 \\
\hline
\end{tabular}

Note. $N=199$.

Main Study. A series of independent t-tests were conducted to see if there was a difference between participants who answered the questions in Nepali and English. There were no significant differences found on means of perceived susceptibility $(t=-.82, d f=195, p=$ $.411)$, perceived severity $(t=-.23, d f=194, p=.806)$, and identified regulation $(t=-1.39, d f=$ $195, p=.165)$. However, the population was found to be different on behavioral intention to drive safely $(t=-2.74, d f=195, p=.007)$. Those who answered in Nepali language intended to drive safely $(M=3.99, S D=.93, n=72)$, more than those who answered the questions in English language $(M=3.64, S D=.82, n=125)$. Hence, language was controlled for the analysis by including it in the path model.

The correlations of the variables in the path analysis are presented in Table 3. Effects coding was used to test the impact of the conditions, such that for autonomy support, strong 
choice message was coded 1 and weak choice message was coded -1 ; and for individualisticcollectivistic dimension, collectivistic message was 1 and individualistic message was -1 . To assess the interaction between the conditions, a variable was created that contrasted strong choice message and collectivistic message (1) vs. weak choice message and individualistic message (-1). Paths that were smaller than .1 were removed. Goodness-of-fit was assessed using the chisquare of the sample correlation, probability associated with the fit, and the root mean square estimate (RMSE) were examined. To allow for re-estimation of the model in post-hoc, modification of indices were also examined. 
Table 3. Summary of Correlations, Means, and Standard Deviations of Variables

\begin{tabular}{|c|c|c|c|c|c|c|c|c|c|c|c|c|c|c|}
\hline & & 1 & 2 & 3 & 4 & 5 & 6 & 7 & 8 & 9 & 10 & 11 & $\mathrm{M}$ & SD \\
\hline 1. & Language & 1 & & & & & & & & & & & & \\
\hline \multirow[t]{2}{*}{2.} & Age & $.280 * *$ & 1 & & & & & & & & & & 20.8 & 2.31 \\
\hline & & & & & & & & & & & & & 7 & \\
\hline 3. & Duration of riding & $.143 *$ & $.394 * *$ & 1 & & & & & & & & & 3.1 & 2.01 \\
\hline \multirow[t]{2}{*}{4.} & Strong Choice & .119 & $.150^{*}$ & .036 & 1 & & & & & & & & 4.27 & .56 \\
\hline & Message & & & & & & & & & & & & & \\
\hline 5. & Collective Message & -.007 & -.029 & -.069 & -.005 & 1 & & & & & & & 4.38 & .61 \\
\hline 6. & Collective X Choice & $-.153 *$ & -.076 & -.087 & -.005 & -.005 & 1 & & & & & & 4.31 & .54 \\
\hline 7. & Identified regulation & .099 & $.144^{*}$ & .023 & -.134 & .007 & .043 & 1 & & & & & 3.77 & .88 \\
\hline \multirow[t]{2}{*}{8.} & Susceptibility & .059 & .077 & .022 & .048 & .120 & -.002 & $.350^{*}$ & 1 & & & & 3.21 & .89 \\
\hline & & & & & & & & $*$ & & & & & & \\
\hline \multirow[t]{2}{*}{9.} & Severity & .018 & .054 & -.043 & -.107 & .033 & .055 & $.350^{*}$ & $.399 *$ & 1 & & & 4.27 & .56 \\
\hline & & & & & & & & $*$ & $*$ & & & & & \\
\hline \multirow[t]{2}{*}{10.} & Behavioral intention & $.193 * *$ & .114 & -.074 & -.071 & -.050 & -.127 & $.225^{*}$ & $.214^{*}$ & $.230 * *$ & 1 & & 4.38 & .61 \\
\hline & & & & & & & & $*$ & $*$ & & & & & \\
\hline \multirow[t]{2}{*}{11.} & Behavior-driving & .208 & .245 & -.099 & .131 & - & -.023 & -.147 & -.087 & -.047 & $.667 * *$ & 1 & 4.31 & .54 \\
\hline & safely & & & & & $.429 * *$ & & & & & & & & \\
\hline
\end{tabular}

Note. ${ }^{*} p<.05 ; * * p<0.01$ level (2-tailed). $N=199$, except for correlations with behavior ( 3 week

lag, $n=37$ ) 
Figure 2. Fit of Hypothesized Path Model Predicting Safe Driving Behavioral Intentions (N $=199)$ and Behavior $(n=37)$

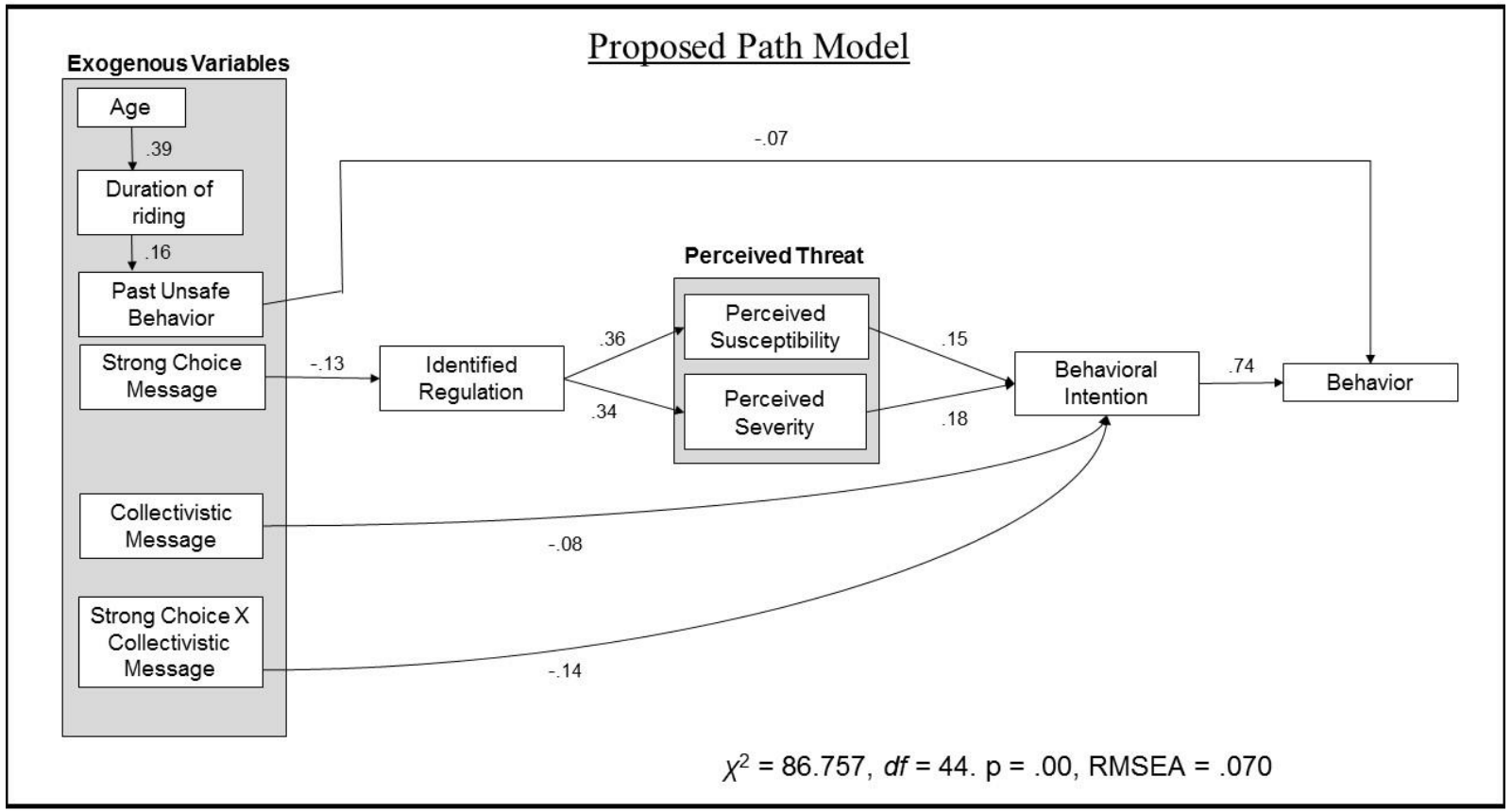

The proposed model (Figure 2) was not a good fit $\left(\chi^{2}=86.757, d f=44, p=.00\right.$; RMSEA $=.07)$. Thus, a series of post-hoc analyses were conducted to find the best fitting model. Missing paths that the program suggested based on the correlations were added to the model. Age was added to the model because of retention bias. The revised model in Figure 3 had an improved fit $\left(\chi^{2}=67.658, d f=52, p=.07, \mathrm{RMSEA}=.04\right)$. 
Figure 3. Revised Path Model Fit, $\mathbf{N}=199$

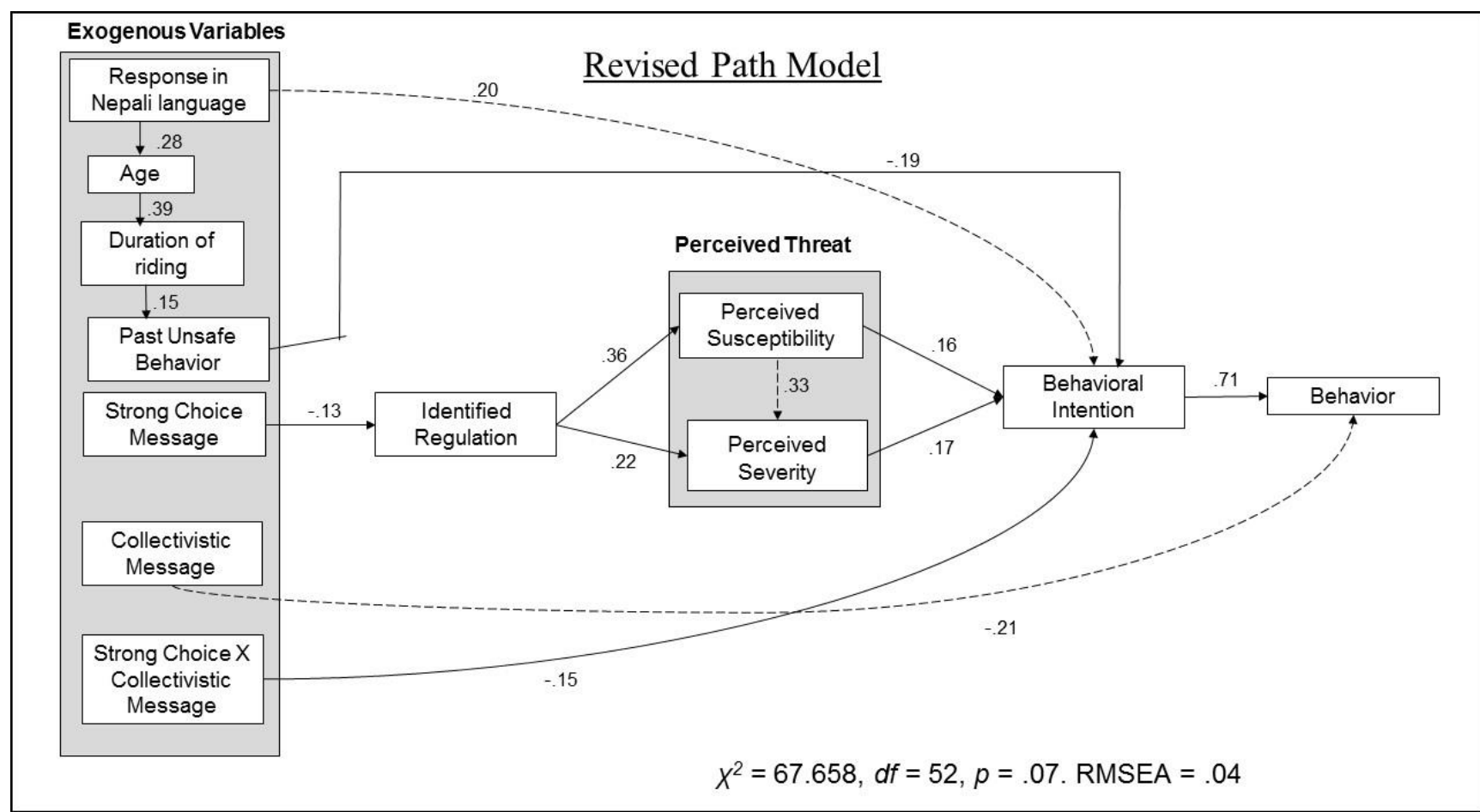

\section{Hypotheses Testing}

H1 predicted that the strong choice message would be positively related to identified regulation. However, this did not hold true. The strong choice message was negatively related to identified regulation $(\rho=-.13, p<.05)$, indicating that participants who viewed the weak choice message were more likely to perceive identified regulation. Hence, the $\mathrm{H} 1$ was not supported.

$\mathrm{H} 2$ predicted that exposure to the collectivistic message would have a positive influence on behavioral intention. The path analysis showed a small negative link between collectivistic message and behavioral intention $(\rho=-.08, p<.05)$. An analyses of missing paths suggested a link between collectivistic message and behavior instead. When the path was included, the path to behavioral intention was no longer significant, hence it was removed from the model. The revised 
model showed collectivistic message was negatively related to behavior $(\rho=-.21, p<.05$; See Figure 3). Hence, H2 was not supported.

H3, which stated that the crossed condition of strong choice message and collectivistic message would be positively related to behavioral intention. However, this hypothesis was not supported $(\rho=-.15, p<.05)$. Analysis of variance showed that crossed condition was marginally significant in influencing behavioral intention $(F(3,197)=3.223, p=.07)$ (See Fig. 5). In examining the means, the condition in which participants viewed the collectivistic and weak choice message had the highest mean on behavioral intention $(M=3.9, S D=.85)$, and the lowest mean on behavioral intention was the group that viewed collectivistic and strong choice message $(M=$ 3.55. $S D=91)$.

In analyzing the interaction further, analyses were conduction in selected conditions. Selecting only cases in the weak choice condition, when behavioral intention is regressed on exposure to the messages, neither collective nor individualistic message had an influence on behavioral intention $(\beta=.078, p=.438, n=99)$. However, in strong choice conditions, individualistic messages predicted behavioral intention with marginal significance $(\beta=-.18, p=$ .087, $n=97$ ) (Exposure to messages were effect coded such that collectivistic appeal was 1 and individualistic appeal message was -1). 
Figure 4. Means of four conditions on behavioral intentions to drive safely

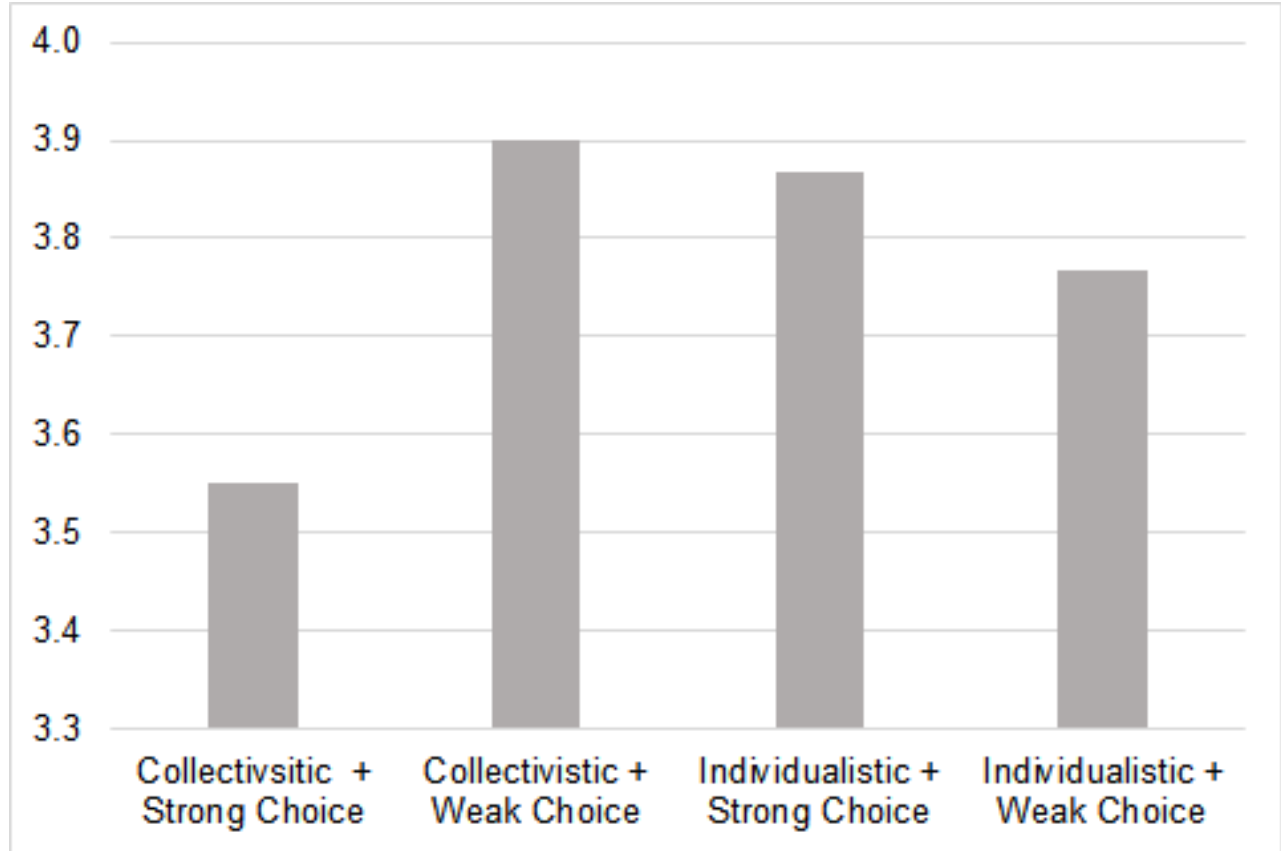

Figure 5. Interaction of conditions on behavioral intention to drive safely

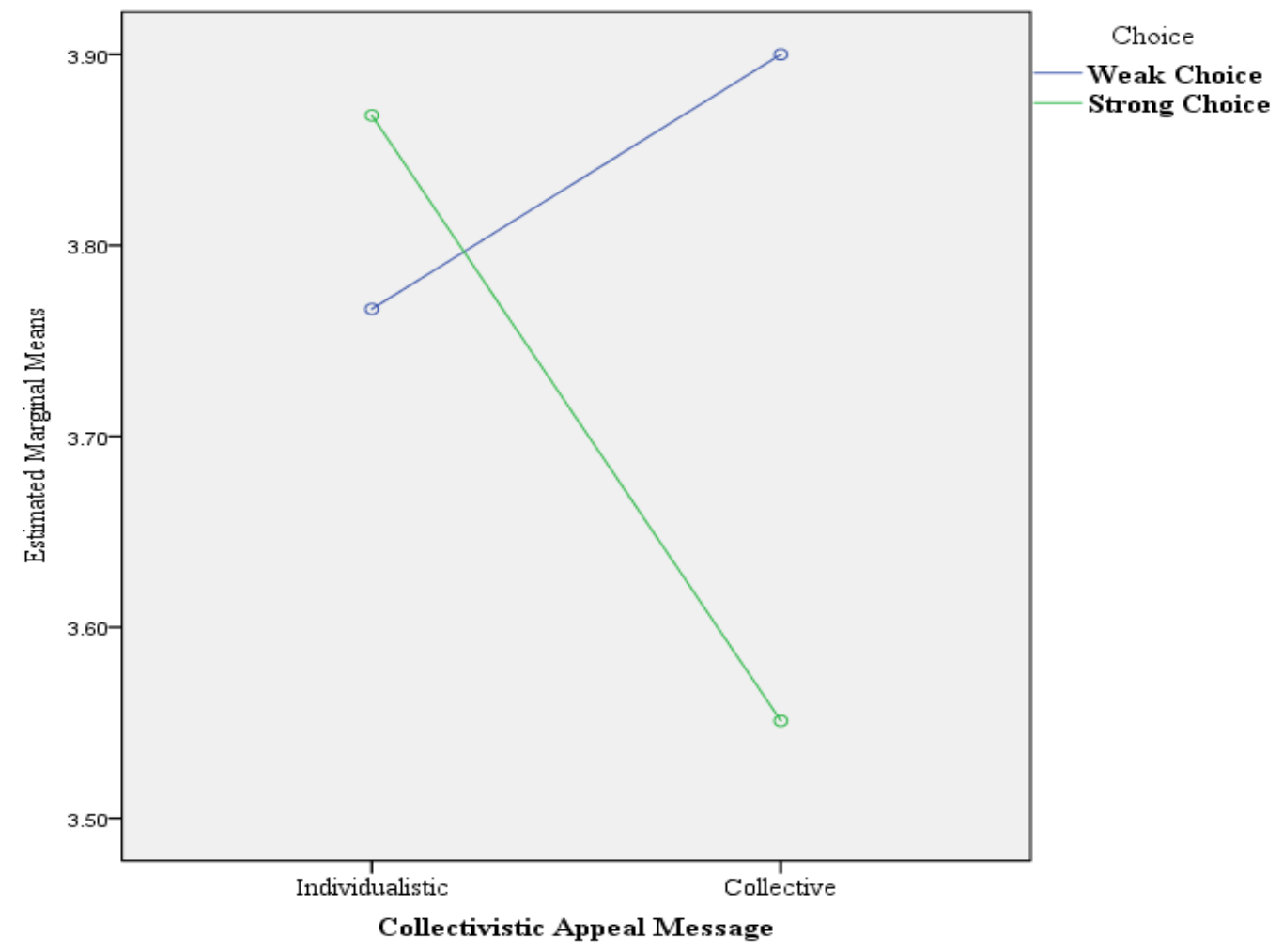


H4 stated that identified regulation will be positively related to perceived susceptibility. The model indicated direct and positive link between identified regulation and perceived susceptibility ( $\rho=.36, p<.05)$, hence H4 was supported. Similarly, identified regulation was positively related to perceived severity $(\rho=.22, p<.05)$, confirming H5.

As predicted by $\mathrm{H} 6$ and $\mathrm{H} 7$, perceived susceptibility $(\rho=.16, p<.05)$ and perceived severity $(\rho=.17, p<.05)$ were positively associated with behavioral intention.

Behavioral intention was strongly associated with behavior $(\rho=.71, p<.00, n=37)$ supporting Hypothesis 14.

Additionally, the analysis indicated paths that were not hypothesized. The model showed path from perceived susceptibility to perceived severity $(\rho=.33, p<.05)$, which was not hypothesized, and age was related to number of years driving/duration of time on the road $(\rho=$ $.39, p<.05)$ and time was related to past unsafe behavior $(\rho=.15, p<.05)$. Also, because behavioral intention scores of individuals who took the survey in Nepali and in English were significantly different, questionnaire language was included in the model. Taking the survey in Nepali was positively related to age $(\rho=.28, p<.00)$ and behavioral intention $(\rho=.20, p<.00)$ (See Figure. 3).

\section{Moderator Hypotheses Testing}

We predicted that perceived barriers would moderate the relationships between perceived susceptibility and behavioral intention (H8), and perceived severity and behavioral intention (H9), such that when perceived barriers are high, the relationship between perceived susceptibility and perceived severity with behavioral intention would be weak, and when perceived barriers are low, the relationship between the independent variables and behavioral intention would be high. Z-scores were created for perceived barrier, perceived severity and 
perceived susceptibility. We created interaction terms for perceived barrier and perceived severity, and for perceived barrier and perceived susceptibility. Behavioral intention was then regressed on the main effects and interaction terms.

As seen in Table 4, the interaction between perceived barriers and perceived susceptibility, and perceived barriers and perceived severity did not significantly predict behavioral intention. Hence, H8 and H9 were not supported.

Table 4. Perceived barriers as moderator of perceived susceptibility and severity on behavioral intention

\begin{tabular}{lccccc}
\hline \hline & \multicolumn{3}{c}{ Behavioral Intention } & & \\
\cline { 2 - 5 } & $B$ & $S E$ & $\beta$ & $T$ & $p$ (Sig.) \\
\cline { 2 - 5 } (Constant) & 2.588 & .494 & & 5.240 & .000 \\
1. Perceived barriers & -.089 & .094 & -.068 & -.942 & .347 \\
2. Perceived susceptibility & .310 & .105 & .214 & 2.942 & .004 \\
3. Interaction barrier x Perceived & .010 & .073 & .010 & .133 & .894 \\
susceptibility & & & & & \\
(Constant) & & & & & \\
1. Perceived barriers & 2.066 & .533 & & 3.875 & .000 \\
2. Perceived severity & -.108 & .093 & -.082 & -1.162 & .247 \\
3. Interaction barrier x Perceived & .445 & .118 & .275 & 3.775 & .000 \\
severity & .095 & .066 & .105 & 1.440 & .151 \\
\hline \hline
\end{tabular}

Note. $N=199$.

Additionally, H10, H11, H12 and H13 predicted that source evaluation variables such as perceived source likability, perceived source dynamism, perceived source competence and perceived source trustworthiness would moderate the relationship between autonomy support 
message and identified regulation such that when the perceived source evaluation variables were high, the relationship between autonomy support message and identified regulation would be strong, and when they are low, the relationship between autonomy support message and identified regulation would be weak. Moderation analysis (using the same techniques as above) found that none of the source credibility variables moderated the relationship between the exposure to autonomy support messages and identified regulation (see Table 5). Hence H10, H11, H12 and H13 were not supported. 
Table 5. Perceived source likability, dynamism, competence and trustworthiness as moderators of choice message on identified regulation

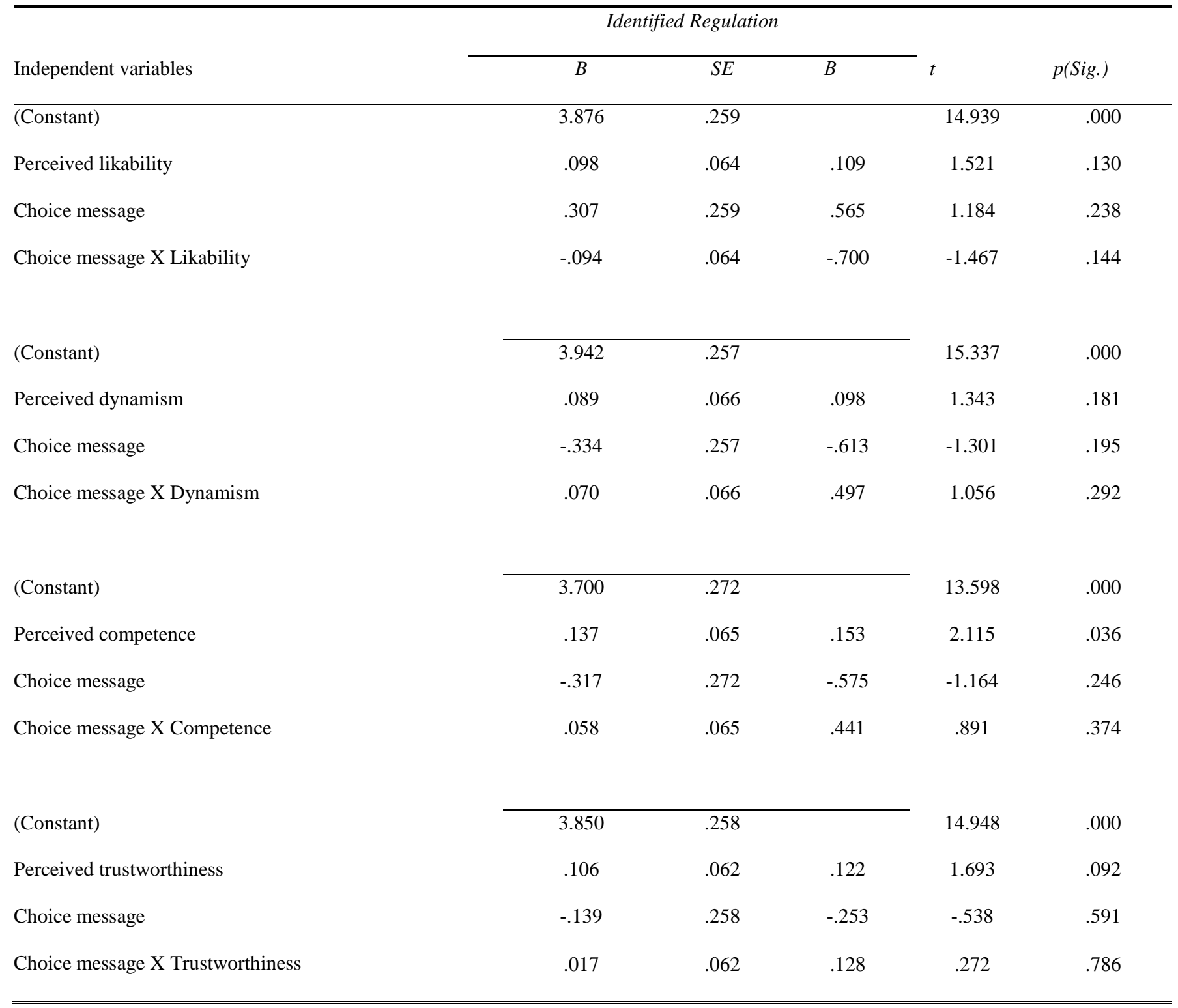

Note. $\mathrm{N}=199$.

H15 predicted that descriptive norm would moderate the relationship between behavioral intention and behavior, and $\mathrm{H} 15$ predicted that injunctive norm would moderate the relationship such that when norms was perceived as high, the relationship between behavioral intention and behavior would be weak, and when low, the relationship between behavioral intention and 
behavior would be strong. No moderator effects were detected (see Table 6). Hence, H14 and H16 were not supported.

A post-hoc analysis showed that although descriptive and injunctive norm did not have direct relationships with behavioral intention and behavior, the norms interacted to influence both dependent variables. An analysis of variance showed the interaction between injunctive and descriptive norm had a stronger influence on behavior $(F=4.61, d f=1.35, p=.02)$ than it did on behavioral intention $(F=1.63, d f=1,35, p=.023)$.

Table 6. Descriptive norm and injunctive norm as moderators of behavioral intention on behavior to drive safely, controlling for age.

\begin{tabular}{|c|c|c|c|c|c|}
\hline & \multicolumn{3}{|c|}{ Behavior } & \multirow[b]{2}{*}{$t$} & \multirow[b]{2}{*}{$p$ (Sig.) } \\
\hline & $B$ & $S E$ & $\beta$ & & \\
\hline (Constant) & -.624 & 1.499 & & -.417 & 680 \\
\hline Age & .004 & .034 & .015 & .110 & .913 \\
\hline Descriptive norm & .055 & .307 & .036 & .179 & .859 \\
\hline Behavioral intention & .898 & .177 & .710 & 5.086 & .000 \\
\hline \multirow[t]{2}{*}{ Interaction behavioral intention $\mathrm{x}$ Descriptive norm } & .101 & .170 & .123 & .597 & .555 \\
\hline & \multicolumn{3}{|c|}{ Behavior } & & \\
\hline (Constant) & -1.169 & .965 & & -1.212 & .235 \\
\hline Age & .028 & .036 & .110 & .776 & .444 \\
\hline Injunctive norm & .278 & .159 & .234 & 1.750 & .090 \\
\hline Behavioral intention & .837 & .168 & .663 & 4.992 & .000 \\
\hline Interaction behavioral intention $\mathrm{x}$ Injunctive norm & .116 & .175 & .089 & .664 & .512 \\
\hline
\end{tabular}

Note: $n=37$. 


\section{Discussion}

Self-determination theory says that an individual is likely to internalize a recommended behavior when he or she identifies value in the recommendation. Although a behavior may not be intrinsically fulfilling, such as indulging is a hobby, a person is likely to engage in a behavior that he or she sees as important for self-preservation, such as using seat belt for protection. This value identification with a behavior is called identified regulation. The theory says that behaviors vary in the degree of being autonomous or controlled (Black \& Deci, 2000). When a behavior is perceived as volitional, or the locus of causality of a behavior is viewed as internal, the behavior is autonomous. On the contrary, when the behavior is performed out of external pressures, the locus of causality is perceived to be controlled by external forces. With autonomous behavior, people are likely experience psychological fulfillment and an increase sense of choice, compared to performing a controlled behavior succumbing to pressures, be it financial or social. Studies of

teacher-student or doctor-patient communication show that when interpersonal interactions allow for autonomy-support - a communication style that emphasize an individual's choice to perform a behavior - people are likely to experience identified regulation, which leads to positive outcomes such as higher performance and preventative health behavior (Black \& Deci, 2000; Williams, Gagne, Ryan, \& Deci, 2002). This study, however, did not find autonomy-support language used in a mediated message triggered identified regulation. In fact, the study found the opposite. Participants identified with the message and perceived the recommended behavior as "the right thing to do" when the source gave them directives, or told them what to do without conveying a sense of choice. Directives have been defined as a form of language where the speaker "expresses an attitude towards a prospective action by the hearer" (Richards \& Schmidt, 1983, p. 39) and the hearer is expected to carry out the action either because of the speaker's 
authority over the hearer, or the speaker's clear desire, in the form of a request, urge or solicitation. In the present study, the directive message uses the construction, "You should..." to tell participants about message sender's injunction regarding the recommended behavior. The finding that participants were more likely to perceive the behavior as important and see value in the recommendation even when messages were in the form of directives is possibly a cultural difference. It can be argued that Nepali culture is marked by high power distance, where authority figures are not questioned and their directives are held in high regards (Anna Perea, 1999; Hofstede, 2001). Additionally, it is noteworthy that Public Service Announcement (PSAs) about serious health issues, such as protection from HIV/AIDS, mostly have an authoritative tone telling the audience what to do, rather than presenting them in autonomy-support language (Johnson, Flora, \& Rimal, 1997). This study therefore suggests that identified regulation is triggered by directives, as opposed to autonomy-support messages, in situations when stakes are perceived to be high and when one's behavior can have a direct and immediate impact on one's health and well-being. Perhaps people are not used to hearing about the dire consequences of a behavior and then being told that it is up to them to make the right choice. Future research should explore if there is an intervention effect when it comes to autonomy-support language's influence on identified regulation. Answers to these questions could mean that the predictions of self-determination theory are conditional.

Further, the results suggest that directives impact risk perception, and this relationship is mediated by identified regulation. In other words, when individuals identify with the message and see value in the recommended behavior, they tended to believe that they were more susceptible to getting into accidents and that the consequences could be severe. This finding is consistent with the health belief model (Janz \& Becker, 1984) that is based on the assumption 
that health behavior depends on the "value one places on a particular goal" (Janz \& Becker, 1984, p. 2). However, the current health belief model does not incorporate the psychological construct of valuing a recommended behavior, rather it makes an assumption that in the context of health, people generally want to be healthy and to avoid being ill/sick or having negative health experience (Champion \& Skinner, 2008b). Hence, this study found that cues to action and the perception of severity and susceptibility is mediated by identified regulation, or the degree of value one places on the recommendation. Measuring the subjective value of the recommended behavior, in addition to the belief about the outcome of the behavior, adds to the current formulation of health belief model. The concept has the potential to explain situations in which people discount public health advice by denying their level of risk, such as youth anti-smoking messages.

The study also tested the persuasive efficacy of messages that appealed to collective versus individual consequences of the behavior. Since Nepal has a collectivistic culture, it was assumed that collectivistic appeal message will influence behavior. On the contrary, the study found that individualistic message had a direct relationship with behavior. That young people in Nepal are motivated by self rather than other-person concerns parallels findings in the U.S. and in Japan, which consistently find that people are motivated by consequences that have a direct impact on their lives (Meares, 2003; Saint-Jacques, 2015; Tyson \& Snyder, 1999).

Additionally, this finding is consistent with a previous cross cultural study that found negative or loss framing of a message paired with individualistic appeal more persuasive than when it was paired with collectivistic appeal (K.-H. Han \& Jo, 2011). This study found that collectivistic appeal message paired with autonomy-support message had the least effect on behavioral intention, compared to other conditions including collectivistic appeal and low 
autonomy-support message. Hence, this finding suggests using individualistic appeal when the health messages highlight potential loss or negative effect of behavior could be most effective. The present study also found individualistic appeals had an effect on behavioral intentions in the low autonomy support (weak choice) condition, but no effect in the high autonomy-support (strong choice) condition. Hence it appears that in the presence of a directive, a message that highlights harm on oneself can influence one's intention, but not when the message highlights one's sense of choice. Perhaps when individuals saw that their actions could harm themselves, it was confusing to receive a message that highlights one's choice to do the right thing. People may interpret messages with a belief that when it comes to actions that could have severe outcome such as losing limbs and even death, there can only be one choice, and that is to follow a recommended behavior; and conversely, if the message is not forceful enough (as in when offered a choice of whether to act or not), they may believe that the consequences are perhaps not so threatening or likely.

It is also noteworthy that the message condition with the least impact on behavioral intentions was the combination of collectivistic appeal and autonomy support (Figure 5). In the absence of either a directive to act or a personal appeal, the Nepali students did not intend to improve their driving.

Additionally, the study predicted that collectivistic appeal messages will influence behavioral intention. Instead, the study found that individualistic appeals had a direct positive impact on behavior. It can thus be interpreted that when individuals see messages that highlight severe harm to oneself, it perhaps requires more time to process the recommended information. Hence, this study suggests that even though individuals may not intend to change behavior immediately, taking time to process and think about the consequences for a while may result in 
behavior change. It is also possible, of course, that an unmeasured factor related to the severe attrition in the dataset for the behavioral follow-up three week after the experiment explains the differences in effects between behavioral intentions and behavior. In any case, this sleeper effect hypothesis - that individual message effects emerged three weeks after exposure - should be empirically tested in future research.

Drawing on intercultural literature, it is important to note that individual differences including personal values and self-construal influence how one perceives messages regardless of the type of culture one comes from (Gudykunst, 1998; Saint-Jacques, 2015).Therefore, future research should examine individual differences as factors for understanding the effects of collectivistic or individualistic appeal messages, instead of assuming homogeneity across culture.

In the context of traffic accident prevention among young people, it may have been critical that the messages showed vivid video of an accident of motorcycle riders taken from a CCTV on a commonly used road in Kathmandu. Young adults find messages with vivid images more effective than vague messages (Lennon \& Rentfro, 2010). Additionally, participants who answered the questions in Nepali and in English languages significantly differed in their intention to drive safely. Those who answered the questions in Nepali intended to drive safely more than those who answered in English. Answering questions in a different language than the language used in the stimulus could contributed to lower intention to follow recommended behavior due to certain language and cultural nuances lost in translation. However, the stimuli seemed to affect behavioral intention across all conditions, this minor confound can be addressed in the future research by making the questionnaire in the same language used in stimulus material. 
As predicted, identified regulation was positively related to both aspects of perceived risk - susceptibility and severity. Thus, when an individual perceives a health recommendation as personally relevant and important, then they are more likely to perceive a greater risk to themselves. The study also found that perceived severity and perceived susceptibility influenced behavioral intention, and that behavioral intentions in turn predicted behavior. These results are consistent with past research (Webb \& Sheeran, 2006).

In this study, the proposed moderators proposed did not hold true. Perceived barriers did not moderate the relationship between perceived susceptibility and behavioral intention, neither did it moderate the relationship between perceived severity and behavioral intention. Regardless of the challenges of driving safely such as unclear traffic rules or non-conducive driving conditions, when people perceive high threat to their health, they are likely to drive safely. Hence, the takeaway from this particular finding is that it is important for public health messages to continue highlighting risks involved with specific behaviors.

The model confirmed strong relationship between behavioral intention and self-reported behavior. However, the prediction that descriptive norms and injunctive norms would moderate the relationship between intention and behavior did not hold true. This finding could have multiple interpretations. A simple way of understanding this would be that when messages are successful in influencing intention, it is inconsequential what the participants' perception of norms are in influencing this specific behavior of riding safely. However, norms literature suggest that descriptive norms influence behavioral intention (Rimal \& Real, 2005), and it can moderate the relationship between intention and actual behavior (Wong \& Sheth, 1985). The failure to find that moderation could be explained through the notion of behavioral attribute, which suggests that certain private behaviors are not as influenced by norms as are social 
behaviors (Rimal \& Lapinski, 2015). Driving a motorcycles could be considered a public behavior but not so much a social one.

Although the prediction of moderation was not supported by the data, a post hoc analysis showed that injunctive norm moderated the relationship descriptive norms and behavior, and descriptive norms and behavioral intention. Analyses showed a stronger effect on behavior than on intention. This finding goes on to show that in this health context, perception of prevalence of the behavior did not influence intention neither behavior, but rather that the two norms together, perceived prevalence and perception of one's society's evaluation of the behavior were important to influence behavior. The implications of this finding are both theoretical and practical. Theoretically, it confirms previous findings of descriptive norm and injunctive norm interact to influence behavior (Rimal \& Lapinski, 2015), and the practical implication is for message designing. If PSA's were to use descriptive norms in messages, it would be effective only if the element of injunctive norms was also included in the message.

Additionally, findings showed age directly linked to the number of years people had been riding, and duration of riding was directly related to past unsafe behavior, stating the obvious that the longer one rode a motorcycle, the more likely they are to indulge in risky behavior. Past unsafe behavior was negatively related to identified regulation, which indicated that participants who had the experience of driving unsafely and admitted to habitually speeding or overtaking dangerously perceived the recommended behavior as less personally important than those who had been safe drivers in the past. These findings cumulatively suggest that traffic safety campaigns should target young adults in schools before they reach the age to ride legally.

Overall, this study has both theoretical and practical implications of understanding health behavior. A construct of identified regulation, borrowed from self-determination theory, extends 
the health belief model beyond its current formulation, contributing to the literature of communication and behavior change. This model can be tested further to understand various other health behavior among young adults such as smoking, drinking, distracted driving, among others.

From message design point of view, this study did not find evidence that autonomysupport language increases one's sense of value in the recommended behavior. On the contrary, messages that told people what to do, instead of giving them a sense of choice, were found to be more effective. Further examination of culture is needed to understand this process. One possible explanation could be that the form of communication (mediated vs. interpersonal) and seriousness of the consequences moderated that relationship. To conclude, for effective intervention and to reduce traffic related accidents, future programs could adopt a mixed approach, of one-to-one counseling to young adults adopting autonomy-support communication, and using directives in mediated messages. Additionally, this study provides further evidence that regardless of cultures, campaigns that target young adults are more effective when persuasive appeal emphasize the outcomes on self (individualistic) rather than the other (collective).

\section{Limitations}

There were a number of limitations to the study. One problem was the large (74\%) percentage of participants who did not complete the 3-week posttest survey assessing the impact of the messages on actual driving behavior. Therefore, in-person, instead of email posttest survey could have yielded less dropouts. Also, providing incentives would have helped participation. Academic credits were not provided because since research participation in exchange for credit 
points is not a general practice in Nepal. Hence, in the future, some financial or material incentive could be provided, which was not used in this study due to budgetary constraints.

Additionally, the questionnaire was translated by a native Nepali speaker, without using the process of translation and back translation, which may have contributed to losing some nuances of the language. For future research, a more colloquial form of the language with rigorous testing is recommended. 


\section{References}

Ajzen, I., \& Fishbein, M. (1980). Understanding attitudes and predicting social behavior. Englewood Cliffs, N.J.: Prentice-Hall Inc.

Ajzen, I., \& Madden, T. J. (1986). Prediction of goal-directed behavior: Attitudes, intentions, and perceived behavioral control. Journal of Experimental Social Psychology, 22(5), 453474. doi:http://dx.doi.org/10.1016/0022-1031(86)90045-4

Anna Perea, M. D. S. (1999). Power distance and collectivist/individualist strategies in alcohol warnings: Effects by gender and ethnicity. Journal of Health Communication, 4(4), 295310. doi:10.1080/108107399126832

Baharudin, H., \& Pandey, S. (2014). Getting over the influence: Crackdown on drinking and driving has lessened road accidents, but public attitude is harder to change. Retrieved from http://nepalitimes.com/article/nation/getting-over-the-influence, 1134

Black, A. E., \& Deci, E. L. (2000). The effects of instructors' autonomy support and students' autonomous motivation on learning organic chemistry: A self-determination theory perspective. Science Education, 84(6), 740-756. doi:10.1002/1098237X(200011)84:6<740::AID-SCE4>3.0.CO;2-3

Burgoon, J. K., Bonito, J. A., Bengsston, B., Cederberg, C., Lundeberg, M., \& Alsspach, L. (2000). Interactivity in human-computer interaction: A study of credibility, understanding, and influence. Computers in Human Behavior, 16, 553-574.

Champion, V. L., \& Skinner, C. S. (2008a). Health Behavior and Health Education : Theory, Research, and Practice (4th Edition). Hoboken, NJ, USA: John Wiley \& Sons.

Champion, V. L., \& Skinner, C. S. (2008b). The Health Belief Model. Hoboken, NJ, USA: John Wiley \& Sons. 
Chatzisarantis, N. L. D., Hagger, M. S., Biddle, S. J. H., Smith, B., \& Wang, J. C. K. (2003). A meta-analysis of perceived locus of causality in exercise, sport, and physical education contexts. Journal of Sports \& Exercise Psychology, 25, 284-306.

Cialdini, R. B. (1994). Influence : the psychology of persuasion. New York: Morrow.

Cialdini, R. B. (2001). Influence : science and practice. Boston, MA: Allyn and Bacon.

Deci, E. L., \& Ryan, R. M. (1985). Intrinsic motivation and self-determination in human behavior. New York, N.Y.: Plemun Press.

Deci, E. L., \& Ryan, R. M. (2012). Self-Determination Theory. In P. A. M. V. Lange, A. W. Kruglanski, \& E. T. Higgins (Eds.), Handbook of Theories of Social Psychology (Vol. 1, pp. 416-437). Thousand Oaks, CA: SAGE Publications Ltd.

Donnell, A. J., Thomas, A., \& Buboltz Jr, W. C. (2001). Psychological Reactance: factor structure and internal consistency of the questionnaire for the measurement of psychological reactance. Journal of Social Psychology, 141(5), 679-687. Retrieved from http://ezproxy.lib.uconn.edu/login?url=http://search.ebscohost.com/login.aspx?direct=tru $\underline{\mathrm{e} \& \mathrm{db}=\mathrm{aph} \& \mathrm{AN}=5662276 \& \text { site }=\text { ehost-live } \& \text { scope }=\text { site }}$

El-Toukhy, S. (2015). Parsing susceptibility and severity dimensions of health risk perceptions. Journal of Health Communication, 20(5), 499-511. doi:10.1080/10810730.2014.989342

Fairchild, A., \& MacKinnon, D. (2009). A general model for testing mediation and moderation effects. Prevention Science, 10(2), 87-99. doi:10.1007/s11121-008-0109-6

Franklin, S. ( 2013). Kathmandu: Traffic hell in Shangri-La. Retrieved from http://pulitzercenter.org/reporting/asia-nepal-kathmandus-traffic-roads-kill-accidentsmotorcycles-stop-lights-taxis-car-fatalities-poverty-economy 
Gibbons, F. X., Gerrard, M., \& Lane, D. J. (2003). A social reaction model of adolescent health risk. In J. Suls \& K. A. Wallston (Eds.), Socail Psychological Foundations of Health and Illness (pp. 107-136). Malden, MA: Blackwell Publishing Ltd.

Gudykunst, W. B. (1998). Editorial. International Journal of Intercultural Relations, 22(2), 107134. doi:http://dx.doi.org/10.1016/S0147-1767(98)00001-7

Hamilton, M. A. (2002). CFA (Version 6.0). Storrs, CT.

Han, K.-H., \& Jo, S. (2011). Does culture matter?: A Cross-national investigation of women's responses to cancer prevention campaigns. Health Care for Women International, 33(1), 75-94. doi:10.1080/07399332.2011.630117

Han, S.-P., \& Shavitt, S. (1994). Persuasion and culture: Advertising appeals in individualistic and collectivistic societies. Journal of Experimental Social Psychology, 30(4), 326. Retrieved from http://ezproxy.lib.uconn.edu/login?url=http://search.ebscohost.com/login.aspx?direct=tru $\underline{\mathrm{e} \& \mathrm{db}=\mathrm{aph} \& \mathrm{AN}=9502275230 \& \text { site }=\text { ehost-live } \& \text { scope }=\text { site }}$

Hass, R. G. (1981). Effects of source characteristics on cognitive responses on persuasion. Hillsdale, NJ: Lawrence Erlbaum Associates.

Hofstede, G. (2001). Culture's consequences : Comparing values, behaviors, institutions, and organizations across nations (2nd ed.). Thousand Oaks, CA: Sage Publications.

Hovland, C. I., \& Weiss, W. (1951). The Influence of source credibility on communication effectiveness. Public Opinion Quarterly, 15(4), 635-650. Retrieved from http://ezproxy.lib.uconn.edu/login?url=http://search.ebscohost.com/login.aspx?direct=tru $\underline{\mathrm{e} \& \mathrm{db}=u f h \& A N=11927286 \& \text { site }=\text { ehost-live } \& \text { scope }=\text { site }}$

Hunter, J., \& Hamilton, M. A. (2002). PATH (Version 6.0). Storrs, CT. 
Janz, N. K., \& Becker, M. H. (1984). The Health Belief Model: A decade later. Health

Education Quarterly, 11(1), 1-47. doi:10.1177/109019818401100101

Jing, Z. (2010). The pursuasiveness of individualistic and collectivistic advertising appeals among chinese generation-x consumers. Journal of Advertising, 39(3), 69-80. doi:DOI 10.2753/JOA0091-3367390305

Johnson, D., Flora, J. A., \& Rimal, R. N. (1997). HIV AIDS Public service announcements around the world: A descriptive analysis. Journal of Health Communication, 2(4), 223234. doi:10.1080/108107397127563

Khaled, R., Biddle, R., Noble, J., Barr, P., \& Fischer, R. (2006). Persuasive interaction for collectivist cultures. Paper presented at the Proceedings of the 7th Australasian User interface conference - Volume 50, Hobart, Australia.

Klein, K., \& Boster, F. (2006). Subjective, descriptive and injunctive norms: Three separate constructs. Paper presented at the International Communication Conference, Dresden International Congress Centre, Dresden, Germany.

Lennon, R., \& Rentfro, R. (2010). Are young adults fear appeal effectiveness ratings explained by fear arousal, perceived threat and perceived efficacy? Innovative Marketing, 6(1), 5865. Retrieved from http://businessperspectives.org/journals_free/im/2010/im_en_2010_01_Lennon.pdf

Meares, M. M. (2003). The new Japan: debunking seven cultural stereotypes: David Matsumoto; Intercultural Press, Yarmouth, Maine, 2002, 236pp. International Journal of Intercultural Relations, 27(3), 375-378. doi:http://dx.doi.org/10.1016/S0147-1767(03)00017-8 
Mishra, B., Sinha(Mishra), N. D., Shukla, S. K., \& Sinha, A. K. (2010). Epidemiological study of road traffic accident cases from Western Nepal. Indian Journal of Community Medicine, 35(1), 115-121. doi:10.4103/0970-0218.62568

Nepal: Road traffic accidents on the rise. (2012). Retrieved from http://www.irinnews.org/report/95960/nepal-road-traffic-accidents-on-the-rise

Pandey, S. (2013). Nepal's highways of death. Nepali Times. Retrieved from http://nepalitimes.com/article/nation/nation-nepal-highways, 622

Parker, D., Manstead, A. S. R., Stradling, S. G., Reason, J. T., \& Baxter, J. S. (1992). Intention to commit driving violations: An application of the theory of planned behavior. Journal of Applied Psychology, 77(1), 94-101. doi:10.1037/0021-9010.77.1.94

Pornpitakpan, C. (2004). The Persuasiveness of Source Credibility: A Critical Review of Five Decades' Evidence. Journal of Applied Social Psychology, 34(2), 243-281. Retrieved from http://ezproxy.lib.uconn.edu/login?url=http://search.ebscohost.com/login.aspx?direct=tru $\underline{\mathrm{e} \& \mathrm{db}=\mathrm{buh} \& \mathrm{AN}=12413425 \& \text { site }=\text { ehost-live } \& \text { scope }=\text { site }}$

Richards, J. C. E., \& Schmidt, R. W. E. (1983). Language and Communication: Longman. Rimal, R. N., \& Lapinski, M. K. (2015). A Re-explication of social norms, ten years later. Communication Theory, 25(4), 393-409. doi:10.1111/comt.12080

Rimal, R. N., Lapinski, M. K., Cook, R. J., \& Real, K. (2005). Moving toward a theory of normative influences: How perceived benefits and similarity moderate the impact of descriptive norms on behaviors. Journal of Health Communication, 10(5), 433-450. doi:10.1080/10810730591009880 
Rimal, R. N., \& Real, K. (2003a). Perceived Risk and Efficacy Beliefs as Motivators of Change. Human Communication Research, 29(3), 370-399. doi:10.1111/j.14682958.2003.tb00844.X

Rimal, R. N., \& Real, K. (2003b). Understanding the influence of perceived norms on behaviors. Communication Theory, 13(2), 184-203.

Rimal, R. N., \& Real, K. (2005). How behaviors are influenced by perceived norms: A test of the Theory of Normative Social Behavior. Communication Research, 32(3), 389-414. doi:10.1177/0093650205275385

Rivis, A., \& Sheeran, P. (2003). Descriptive norms as an additional predictor in the theory of planned behaviour: A meta-analysis. Current Psychology, 22(3), 218-233. doi:10.1007/s12144-003-1018-2

Ryan, R. M., \& Connell, J. P. (1989). Perceived locus of causality and internalization: Examining reasons for acting in two domains. Journal of Personality and Social Psychology, 57(5), 749-761. doi:10.1037/0022-3514.57.5.749

Ryan, R. M., \& Deci, E. L. (2000). Self-determination theory and the facilitation of instrinsic motivation, social development, and well-being. American Pyschologist, 55(1), 68-78. doi:10.1037/0003-066X.55.1.68

Saint-Jacques, B. (2015). Intercultural Communication in a Globalized World. In L. A. Samovar, R. E. Porter, E. R. McDaniels, \& C. S. Roy (Eds.), Intercultural Communcation: A Reader (14 ed., pp. 16-26). Boston, MA: Cengage Learning.

Thapa, A. J. (2013). Road Safety Status of Nepal 2013. Retrieved from http://www.unece.org/fileadmin/DAM/trans/doc/2013/wp1/newdelhi/New_Delhi_dec_20 13_Nepal.pdf 
Tyson, C. B., \& Snyder, L. B. (1999). The Impact of Direct Mail Video. Public Relations Quarterly, 44(1), 28-32. Retrieved from http://ezproxy.lib.uconn.edu/login?url=http://search.ebscohost.com/login.aspx?direct=tru $\underline{\mathrm{e} \& \mathrm{db}=u f h \& A N=1804332 \& \text { site }=\text { ehost-live } \& \text { scope }=\text { site }}$

Urban mobility in Kathmandu: Status and trends. Retrieved from

Valley's traffic unbridled chaos. (2011). New Spotlight. Retrieved from http://www.spotlightnepal.com/News/Article/VALLEY\%E2\%80\%99S-TRAFFIC$\underline{\text { Unbridled-Chaos- }}$

Webb, T. L., \& Sheeran, P. (2006). Does changing behavioral intentions engender behavior change? A meta-analysis of the experimental evidence. Psychol Bull, 132(2), 249-268. doi:10.1037/0033-2909.132.2.249

Williams, G. C., Gagne, M., Ryan, R. M., \& Deci, E. L. (2002). Facilitating autonomous motivation for smoking cessation. Health Psychology, 21(1), 40-50. doi:10.1037/02786133.21.1.40

Williams, G. C., Niemiec, C. P., Patrick, H., Ryan, R. M., \& Deci, E. L. (2009). The importance of supporting autonomy and perceived competence in facilitating long-term tobacco abstinence. Annals of Behavioral Medicine, 37(3), 315-324. doi:10.1007/s12160-009$9090-y$

Williams, G. C., Rodin, G. C., Ryan, R. M., Grolnick, W. S., \& Deci, E. L. (1998). Autonomous regulation and long-term medication adherence in adult outpatients. Health Psychology, 17(3), 269-276. doi:10.1037/0278-6133.17.3.269 
Witte, K., Cameron, K. A., McKeon, J. K., \& Berkowitz, J. M. (1996). Predicting Risk Behaviors: Development and Validation of a Diagnostic Scale. Journal of Health Communication, 1(4), 317-342. doi:10.1080/108107396127988

Wong, J. K., \& Sheth, J. N. (1985). Explaining intention-behavior discrepancy - A paradigm. Advances in Consumer Research, 12(1), 378-384. Retrieved from http://ezproxy.lib.uconn.edu/login?url=http://search.ebscohost.com/login.aspx?direct=tru $\underline{\mathrm{e} \& \mathrm{db}=\mathrm{bth} \& \mathrm{AN}=6430998 \& \text { site }=\text { ehost-live } \& \text { scope }=\text { site }}$ 